\title{
Numerical multiscale modelling of nonlinear elastowetting
}

\author{
Riccardo De Pascalis ${ }^{\mathrm{a}, \mathrm{b}, *}$, Julien Dervaux ${ }^{\mathrm{a}}$, Ioan Ionescu ${ }^{\mathrm{b}, \mathrm{c}}$, Laurent Limat ${ }^{\mathrm{a}}$ \\ ${ }^{a}$ MSC, Université Paris Diderot, CNRS UMR 705\%, Sorbonne Paris Cité, \\ 10 Rue A. Domon et L. Duquet, F-75013 Paris, France \\ ${ }^{\mathrm{b}}$ LSPM, Université Paris 13, CNRS UPR 3407, Sorbonne Paris Cité, \\ 99 Avenue J.-B. Clement, F-93430 Villetaneuse, France \\ ${ }^{\mathrm{c}}$ IMAR, Romanian Academy, Bucharest, Romania
}

\begin{abstract}
We investigate here the static finite deformation of two (or more) three-dimensional nonlinear elastic solids which merged in a third medium, define a triple line (TL). The total energy, accounting for elastic, surface and possible gravity potentials is then minimized numerically in order to solve two physical problems of interest: (i) a soft incompressible axisymmetric drop at rest on a stiffer substrate and (ii) a stiff drop at rest upon a softer substrate, both in situations of strong substrate surface tension effects. Because of the interplay between surface and bulks properties, the behavior of the TL is related to another smaller length-scale (typically micrometre scale) than the elastic solids lengthscale (typically millimetre scale). A unified deformability parameter relating all scales is introduced and a multi-scale discretization of the domain is used.

A first example approached by our model is the elasto-wetting problem in which a very soft drop sits on a stiffer elastic solid. The numerical predictions of our model are compared with the analytical results of the linear elastic theory. In particular, our computations show that the Laplace pressure, together with the asymmetry of the solid surface tensions (between the wet and dry part of the interface), account for the rotation of the cusp of the ridge.

In the second example, motivated by indentation problems of a nonlinear elastic substrate by a stiff sphere, we analyse the interplay of the solid surface tensions and elasticity at a small-scale. The numerical results also show that gravity might have an important effect and must be included in any micro-scale modelling.
\end{abstract}

KEYWORDS: elastowetting, triple line (TL), surface tension, multiscale modelling, nonlinear elasticity, finite elements

\section{Introduction}

Soft elastic solids can be deformed by surface effects ([Roman and Bico, 2010]) in a fashion similar to that of fluids ([De Gennes et al., 2013]). While capillary forces are proportional to a certain characteristic length $L_{\mathrm{c}}$, elastic forces are proportional to its square $L_{\mathrm{c}}^{2}$, such that, below some lenghtscale, the capillary forces becomes dominant and can no longer be neglected. Elasto-capillarity focuses on all phenomena arising from the coupling between bulk elastic deformations with surface effects. Because capillary forces tend to minimize the surface area, or interface, between two media in different thermodynamic phases (such as the interface between a solid and the air or between a solid and a liquid), surface effects act as external loads. For example, [Mora et al., 2013] showed experimentally that a centimetre-size soft elastic solid immersed in a liquid can undergo large deformations, stretching, shortening and bending its slender structure, or rounding as much as bubbles. More recently, [Mora and Pomeau, 2015] investigated the deformation of a sharp soft solid edge (hydrogel) surrounded by a liquid, and showed how the sharp edges round under the effect of surface tensions. Also, several instabilities due to surface effects in elastic solids have been explored by [Mora et al., 2010, Ben Amar and Ciarletta, 2010, Mora et al., 2011, Dervaux et al., 2011, Dervaux and Ben Amar, 2011, Ciarletta, 2013, Taffetani and Ciarletta, 2015].

\footnotetext{
${ }^{*}$ Author for correspondence: riccardo.depascalis@gmail.com
} 
Motivated by fundamental questions as well as problems of practical importance in fluid handling applications ([Unger et al., 2000, Boland et al., 2006, Sokuler, 2010, Chung, 2011]) a lot of interest has been devoted recently to elastowetting problems where, typically, liquid drops are in contact with both the air and a soft solid. In such problems, three thermodynamic phases (typically a solid a liquid and its vapour) are in contact and the intersection between these interfaces defines the so-called triple line (TL). The wetting of these soft viscoelastic materials differs from that on rigid substrates where the (static) behavior of the TL is solely controlled by surface tensions, leading to the so-called Young's equation (see formula (28)). In elastowetting however, the vertical component of the capillary forces induce the formation of a ridge on the solid surface beneath the TL $([\mathrm{Yu}, 2012])$ and the competition between elasticity and capillarity must be taken into account to describe the experimental observations. Despite a large literature starting in the sixities ([Lester, 1961, Rusanov, 1975, Shanahan and De Gennes, 1987, Shanahan, 1987b, Shanahan, 1987a]) up to more recent works ([Pericet-Camara et al., 2009, Jerison et al., 2011, Lubarda, 2012, Style and Dufresne, 2012, Limat, 2012, Nadermann et al., 2013, Style et al., 2013a, Dervaux and Limat, 2015, Bostwick et al., 2014] and references therein), several outstanding questions are still debated and a large scientific community is currently investigating both static and dynamic effects in elastowetting. For example, the detailed structure of the soft elastic ridge beneath the TL ([White, 2003, Marchand et al., 2012b, Park et al., 2014, Lubbers et al., 2014]) is still not resolved in the general case of asymmetric surface tensions. On the theoretical side, the vast majority of studies on elastowetting have been developed within the framework of linear elasticity, although with a few notable exceptions confined to particular cases ([Madasu and Cairncross, 2004, Yu and Zhao, 2009]), and nonlinear effects, either geometric or material, have yet to be incorporated within a theoretical framework. [Lubbers et al., 2014], in their final discussion, include nonlinearities as one of next major challenge for the field.

Since the work of [Shuttleworth, 1950], surface effects arising in solids have been, in general, considered differently from those arising in liquids, distinguishing between surface energy (work necessary to form unit area of surface by a division process) and surface tension (tangential stress (force per unit length) in the surface layer), while for liquids no distinction is needed. This topic has been recently investigated by [Marchand et al., 2012a] and [Weijs et al., 2013] for example. In this paper, we focus on the effects of material and geometric nonlinearities on the TL and we leave aside the possible differences between surface tension and surface energy, taking the surface tension and the surface energy as equivalents ([Mora et al., 2013]). These forces, here, will be called 'surface tensions'. Moreover, for the applications given in this paper we will consider incompressible isotropic materials, hence misleading interpretations for the surface effects can be avoided (see [Weijs et al., 2013]).

Modelling TL is a challenge for any continuum model because of geometrical and material nonlinearities, discontinuities in the loading surface forces where pointwise forces produce high values of the deformation gradient, as well as the existence of multiple scales. To tackle this problem, we develop here a variational approach to overcome some of the existing difficulties. Under the isotropy and hyperelasticity assumption, the elastic energy density used to describe the solid response is chosen to be of the Mooney-Rivlin type. The total energy of the system, consists of an elasto-capillarity energy (which includes elastic and superficial energies), as well as the gravitational potential energy. This last contribution, even at very small scales, could have a non negligible effect for very soft materials such as those typically used in micro-fabricated devices ([Xu et al., 2013]). The static equilibrium is a stationary point of the total energy, and since the equation obtained from the minimization process is unlikely solvable analytically, a standard Newton-Raphson procedure is adapted here for Finite Element Method (FEM) simulations in the case of pinned contact line. However, the presence of several bulks and interface properties leads to physical effects occurring at different scales. In order to properly model the TL, all these scales must be taken into account. Here, a unified deformability parameter $\delta$ is used to relate all the scales and a multiscale discretization of the domain is employed in order to capture phenomena occurring at different scales and thus the global behavior of the system. The displacement field is then calculated step by step, iterating the algorithm and increasing the deformability parameter starting from $\delta=0$.

In order to gain physical insights into the behavior of the TL, we focus on two axisymmetric problems: (i) firstly, motivated by elastowetting problems, we study the behavior of a very soft drop on a stiffer 
nonlinear elastic substrate. In particular, we investigate the limiting case of a drop with a vanishing shear modulus that has a purely hydrostatic stress and thus behaves as a fluid at rest. The numerical results are presented and discussed for both the symmetric and asymmetric cases (i.e. identical or different solid surfaces tensions between the dry and wetted parts). In the symmetric case, our numerical results at small $\delta$ are compared to a linear theory ([Bostwick et al., 2014]) which uses a displacement potential function in a small-deformations framework. The good agreement between analytics and numerics validate the numerical method. For higher liquid-air surface tensions or softer substrate however, very large deformations occur and the linear theory fails. In the asymmetric case, we predict a rotation of the ridge at the TL, a fact which is also observed experimentally and a nonlinear response of its height with respect to the substrate's thickness. (ii) In a second part, motivated by indentation problems, ([Style et al., 2013b, Xu et al., 2014, Cao et al., 2014]), we investigate the case of a stiff elastic sphere on a softer substrate. A method similar to that of the first application is used in the analysis and in the numerical computations. We show that, gravity might have an important effect on the behavior of the TL and must be included in any indentation micro-scale modelling which deal, for example, with very soft materials.

The structure of our paper is as follows: in section 2, notations, definitions of the total energy of the system, kinematics and constitutive assumptions are introduced for a general system of two isotropic hyperelastic compressible bodies which are statically in equilibrium under the effect of superficial forces and gravity effects. In section 3 a minimization procedure for the total energy in 3-D is derived. Sections 4 and 5 analyse and discuss two applications, under the axisymmetry and incompressibility assumptions: the case of a soft drop on a stiffer substrate and the stiff drop on the softer substrate. Finally, conclusions are presented in the section 6.

\section{Elastocapillary energy, kinematics and constitutive assumptions}

Two solids $\mathcal{S}_{1}, \mathcal{S}_{2}$ and a vapour $\mathcal{V}$ are described, in the current three-dimensional Euclidean space, by the regions $\tilde{\mathcal{D}}_{1}, \tilde{\mathcal{D}}_{2}$ and $\tilde{\mathcal{D}}_{\mathrm{V}}{ }^{1}$, respectively. The elastic domain of interest in the Eulerian configuration is $\tilde{\mathcal{D}}=\tilde{\mathcal{D}}_{1} \cup \tilde{\mathcal{D}}_{2}$, while the interface between $\mathcal{S}_{1}$ and $\mathcal{S}_{2}$ is denoted by $\tilde{\Gamma}_{12}$, between $\mathcal{S}_{1}$ and $\mathcal{V}$ by $\tilde{\Gamma}_{1 \mathrm{~V}}$ and between $\mathcal{S}_{2}$ and $\mathcal{V}$ by $\tilde{\Gamma}_{2 \mathrm{~V}}$, respectively, and shortly all gathered by $\tilde{\Gamma}=\tilde{\Gamma}_{12} \cup \tilde{\Gamma}_{1 \mathrm{~V}} \cup \tilde{\Gamma}_{2 \mathrm{~V}}$. $\tilde{\Lambda}$ denotes all other interfaces, where conditions are imposed on the displacement field. The introduced geometrical terms refer to the current configuration, and the same notations with the superscript 0 are used for the reference configuration $\tilde{\mathcal{D}}^{0}$.

Surface effects are considered by assuming the presence of a piecewise constant, in magnitude, surface tension $\tilde{\gamma}$ on $\tilde{\Gamma}$, such that

$$
\tilde{\gamma}=\left\{\begin{array}{l}
\tilde{\gamma}_{12} \text { on } \tilde{\Gamma}_{12} \\
\tilde{\gamma}_{1 \mathrm{~V}} \text { on } \tilde{\Gamma}_{1 \mathrm{~V}} \\
\tilde{\gamma}_{2 \mathrm{~V}} \text { on } \tilde{\Gamma}_{2 \mathrm{~V}} .
\end{array}\right.
$$

Assuming hyperelasticity, the internal energy of the system is the sum of the potential elastic energy (strain energy function) $\tilde{W}_{\mathrm{e}}$ and the surface potential energy $\tilde{W}_{\mathrm{s}}=\tilde{\gamma}$. Effects from the gravity $\boldsymbol{g}$ there will be also taken into account so that the total potential energy, denoted by $\tilde{\Pi}$, is

$$
\tilde{\Pi}=\tilde{\mathcal{I}}_{\mathrm{e}}+\tilde{\mathcal{I}}_{\mathrm{s}}-\tilde{\mathcal{I}}_{\mathrm{g}}
$$

where

$$
\tilde{\mathcal{I}}_{\mathrm{e}}=\int_{\tilde{\mathcal{D}}^{0}} \tilde{W}_{\mathrm{e}} \mathrm{d} \tilde{\mathcal{D}}^{0}, \quad \tilde{\mathcal{I}}_{\mathrm{s}}=\int_{\tilde{\Gamma}} \tilde{W}_{\mathrm{s}} \mathrm{d} \tilde{\Gamma}, \quad \tilde{\mathcal{I}}_{\mathrm{g}}=\int_{\tilde{\mathcal{D}}^{0}} \tilde{\rho}_{0} \tilde{\boldsymbol{g}} \cdot \tilde{\boldsymbol{u}} \mathrm{d} \tilde{\mathcal{D}}^{0},
$$

and where $\tilde{\boldsymbol{u}}$ denotes the displacement vector field.

In order to obtain a dimensionless formulation of the problem, let us introduce the characteristic length $L_{\mathrm{c}}$, which allows to define the dimensionless domains $\mathcal{D}, \mathcal{D}_{1}, \mathcal{D}_{2}$, the dimensionless interfaces $\Gamma$ and $\Lambda$ and the dimensionless displacement $\boldsymbol{u}$

$$
\tilde{\mathcal{D}}=L_{\mathrm{c}} \mathcal{D}, \quad \tilde{\mathcal{D}}_{i}=L_{\mathrm{c}} \mathcal{D}_{i}(i=1,2), \quad \tilde{\Gamma}=L_{\mathrm{c}} \Gamma, \quad \tilde{\Lambda}=L_{\mathrm{c}} \Lambda, \quad \tilde{\boldsymbol{u}}=L_{\mathrm{c}} \boldsymbol{u},
$$

\footnotetext{
${ }^{1}$ The upper symbol ${ }^{\sim}$ will be always used to denote dimensional quantities.
} 
and let $\gamma_{\mathrm{c}}$ be the characteristic surface tension which gives the dimensionless surface energy $W_{\mathrm{s}}$ and the surface tension $\gamma$,

$$
\tilde{W}_{\mathrm{s}}=\gamma_{\mathrm{c}} W_{\mathrm{s}}, \quad \tilde{\gamma}=\gamma_{\mathrm{c}} \gamma .
$$

Let also denote by $\Sigma_{\mathrm{c}}$ the characteristic stress, which gives the dimensionless strain energy function $W_{\mathrm{e}}$ and the two dimensionless (infinitesimal) shear modulus $\mu_{i}$ of $S_{i}$

$$
\tilde{W}_{\mathrm{e}}=\Sigma_{\mathrm{c}} W_{\mathrm{e}}, \quad \tilde{\mu}_{i}=\Sigma_{\mathrm{c}} \mu_{i},(i=1,2) .
$$

Finally, denoting by $\rho_{\mathrm{c}}$ and by $g_{\mathrm{c}}$ the characteristic density and the characteristic gravity, we can define

$$
\tilde{\rho}_{0}=\rho_{\mathrm{c}} \rho_{0}, \quad \tilde{\boldsymbol{g}}=g_{\mathrm{c}} \boldsymbol{g},
$$

the dimensionless density and the gravity vector.

Since we deal with several bulks (and thus several elastic coefficients) and several interfaces (and thus several surface tensions), several lengthscales, which relate surface tensions to the elastic effects, appear. These physical lengthscales have to be considered in addition to the characteristic length $L_{\mathrm{c}}$, which describe the characteristic size of the solids. In fact, the ratios

$$
l_{\mathrm{s} 1}=\frac{\tilde{\gamma}_{1 \mathrm{~V}}}{\mu_{1} \Sigma_{c}}, \quad l_{\mathrm{s} 2}=\frac{\tilde{\gamma}_{2 \mathrm{~V}}}{\mu_{2} \Sigma_{c}}
$$

define two independent elastocapillary length scales for each solid $\mathcal{S}_{1}$ and $\mathcal{S}_{2}$. Since the TL is related to both (or more) solids, it is clearly related to both length scales $l_{\mathrm{s} 1}$ and $l_{\mathrm{s} 2}$. Choosing one characteristic elastocapillary length we introduce a unified non-dimensional parameter, called here the deformability parameter $\delta$, which combines the different scales involved in the modelling of the TL and the dimensionless gravity parameter $\eta$, which accounts for the gravity effects

$$
l_{\mathrm{s}}=\frac{\gamma_{\mathrm{c}}}{\Sigma_{\mathrm{c}}}, \quad \delta=\frac{l_{\mathrm{s}}}{L_{\mathrm{c}}}=\frac{\gamma_{\mathrm{c}}}{L_{\mathrm{c}} \Sigma_{\mathrm{c}}}, \quad \eta=\frac{L_{\mathrm{c}} \rho_{\mathrm{c}} g_{\mathrm{c}}}{\Sigma_{\mathrm{c}}},
$$

Both parameters $\delta$ and $\eta$ controls the overall deformation.

The total potential dimensionless energy $\Pi$ for $\mathcal{D}$ can be therefore rewritten in a dimensionless form as

$$
\Pi(\boldsymbol{u})=\mathcal{I}_{\mathrm{e}}(\boldsymbol{u})+\mathcal{I}_{\mathrm{s}}(\boldsymbol{u})-\mathcal{I}_{\mathrm{g}}(\boldsymbol{u})
$$

where

$$
\mathcal{I}_{\mathrm{e}}(\boldsymbol{u})=\int_{\mathcal{D}^{0}} W_{\mathrm{e}}(\boldsymbol{u}) \mathrm{d} \mathcal{D}^{0}, \quad \mathcal{I}_{\mathrm{s}}(\boldsymbol{u})=\delta \int_{\Gamma} W_{\mathrm{s}} \mathrm{d} \Gamma, \quad \mathcal{I}_{\mathrm{g}}(\boldsymbol{u})=\eta \int_{\mathcal{D}^{0}} \boldsymbol{g} \cdot \boldsymbol{u} \mathrm{d} \mathcal{D}^{0} .
$$

In the isotropic case, the potential elastic energy density $W_{\mathrm{e}}$ is related to the elastic stress by the following nonlinear relationship,

$$
\boldsymbol{\sigma}=\alpha_{0} \mathbf{I}+\alpha_{1} \mathbf{B}+\alpha_{2} \mathbf{B}^{2},
$$

where $\alpha_{j}=\alpha_{j}\left(I_{1}, I_{2}, I_{3}\right), j=0,1,2$, are the elastic response functions defined by

$$
\alpha_{0}=2 \sqrt{I_{3}} W_{\mathrm{e}, 3}, \quad \alpha_{1}=\frac{2}{\sqrt{I_{3}}}\left[W_{\mathrm{e}, 1}+I_{1} W_{\mathrm{e}, 2}\right], \quad \alpha_{2}=-\frac{2}{\sqrt{I_{3}}} W_{\mathrm{e}, 2},
$$

where $W_{\mathrm{e}, i}=\partial W_{\mathrm{e}} / \partial I_{i}$ and

$$
I_{1}=\operatorname{tr} \mathbf{B}, \quad I_{2}=\frac{1}{2}\left[(\operatorname{tr} \mathbf{B})^{2}-\operatorname{tr} \mathbf{B}^{2}\right], \quad I_{3}=J^{2}=\operatorname{det} \mathbf{B},
$$

are the principal invariants of $\mathbf{B}$, where $\mathbf{B}$ is the left Cauchy-Green tensor $\mathbf{F F}^{\mathrm{T}}$ and $\mathbf{F}=\mathbf{F}(\boldsymbol{u})=\mathbf{I}+\nabla \boldsymbol{u}$ is the deformation gradient $F_{i j}=\partial x_{i} / \partial X_{j}$. Here $\boldsymbol{x}$ and $\boldsymbol{X}$ denote the coordinate systems in the reference and current configuration, respectively, which are related by $\boldsymbol{x}=\boldsymbol{X}+\boldsymbol{u}$. In the incompressible limit, the dimensionless Cauchy stress tensor (12) simplifies to:

$$
\boldsymbol{\sigma}=-p \mathbf{I}+2\left[W_{1}+I_{1} W_{2}\right] \mathbf{B}-2 W_{2} \mathbf{B}^{2}
$$


where $\mathrm{p}$ is the Lagrange multiplier introduced by the incompressibility constraint. For future uses, the first Piola-Kirchhoff stress tensor $\mathbf{P}$ and the Kirchhoff stress tensor $\boldsymbol{\tau}_{K}$, are also recalled,

$$
\mathbf{P}=J \boldsymbol{\sigma} \mathbf{F}^{-\mathrm{T}}, \quad \boldsymbol{\tau}_{K}=J \boldsymbol{\sigma} .
$$

In order to write the total energy of the system (10) in the reference configuration, the superficial contribution of the energy can be pulled-back, which thanks to Nanson's formula $\boldsymbol{n} \mathrm{d} \Gamma=J \mathbf{F}^{-\mathrm{T}} \boldsymbol{n}^{0} \mathrm{~d} \Gamma^{0}$ (here $\boldsymbol{n}$ and $\boldsymbol{n}^{0}$ are the outward unit normals in the deformed and undeformed configurations), can be rewritten as

$$
\mathcal{I}_{\mathrm{s}}(\boldsymbol{u})=\delta \int_{\Gamma^{0}} W_{\mathrm{s}} J\left\|\mathbf{F}^{-\mathrm{T}}(\boldsymbol{u}) \boldsymbol{n}^{0}\right\| \mathrm{d} \Gamma^{0} .
$$

\section{Minimization of the total energy: Euler-Lagrange equations}

The displacement solution $\boldsymbol{u}$ is a stationary point of the total energy (10), i.e. a solution for the EulerLagrange equation:

$$
\Pi^{\prime}(\boldsymbol{u})(\boldsymbol{v})=\frac{\mathrm{d}}{\mathrm{d} t} \Pi(\boldsymbol{u}+t \boldsymbol{v})_{\mid t=0}=0,
$$

for every $\boldsymbol{v}$ test function. Standard calculations show that

$$
\begin{gathered}
\mathcal{I}_{\mathrm{e}}^{\prime}(\boldsymbol{u})(\boldsymbol{v})=\int_{\mathcal{D}^{0}} \mathbf{P}(\boldsymbol{u}): \nabla \boldsymbol{v} \mathrm{d} \mathcal{D}^{0}=\int_{\mathcal{D}^{0}} \boldsymbol{\tau}_{K}(\boldsymbol{u}): \nabla \boldsymbol{v} \mathbf{F}^{-1}(\boldsymbol{u}) \mathrm{d} \mathcal{D}^{0}, \\
\mathcal{I}_{\mathrm{s}}^{\prime}(\boldsymbol{u})(\boldsymbol{v})=\int_{\Gamma^{0}} \delta \gamma \mathcal{G}(\boldsymbol{u}, \boldsymbol{v}) \mathrm{d} \Gamma^{0},
\end{gathered}
$$

where

$$
\mathcal{G}(\boldsymbol{u}, \boldsymbol{v})=J\left\|\mathbf{F}^{-\mathrm{T}} \boldsymbol{n}^{0}\right\| \mathbf{F}^{-T}: \boldsymbol{\nabla} \boldsymbol{v}-\frac{\left(\mathbf{F}^{-\mathrm{T}} \boldsymbol{\nabla} \boldsymbol{v} \mathbf{F}^{-\mathrm{T}}\right) \boldsymbol{n}^{0} \cdot \mathbf{F}^{-\mathrm{T}} \boldsymbol{n}^{0}}{\left\|\mathbf{F}^{-\mathrm{T}} \boldsymbol{n}^{0}\right\|}
$$

where in order to simplify notations (here and in the following where no confusion occurs) the $\boldsymbol{u}$ dependence of the deformation gradient $\mathbf{F}=\mathbf{F}(\boldsymbol{u})$ has been omitted, and

$$
\mathcal{I}_{\mathrm{g}}^{\prime}(\boldsymbol{u})(\boldsymbol{v})=\int_{\mathcal{D}^{0}} \eta \boldsymbol{g} \cdot \boldsymbol{v} \mathrm{d} \mathcal{D}^{0},
$$

for $\boldsymbol{u}-\boldsymbol{u}^{\mathbf{0}} \in \mathcal{U}$ and for every $\boldsymbol{v} \in \mathcal{U}$, where

$$
\mathcal{U}=\left\{\boldsymbol{v} \in W^{1, s}\left(\mathcal{D}^{0}, \mathbb{R}^{3}\right) ; \boldsymbol{v}_{\mid \Lambda^{0}}=\mathbf{0}\right\},
$$

and $\boldsymbol{u}^{0}$ is some displacement field imposed on the boundary $\Lambda^{0}$, and $W^{1, s}\left(\mathcal{D}^{0}, \mathbb{R}^{3}\right)$ denotes the Sobolev space of vectorial functions defined on $\mathcal{D}^{0}$, with $s \geq 1$.

Generally, equation (18) cannot be evaluated analytically and a numerical scheme is necessary. One of the most popular procedure is the so called Newton-Raphson method (see for instance [Le Tallec, 1994]), which is presented below. Let us consider a sequence $\boldsymbol{u}_{n}$ s.t. $\boldsymbol{u}_{n}-\boldsymbol{u}^{0} \in \mathcal{U}, \boldsymbol{u}_{n+1}=\boldsymbol{u}_{n}+\boldsymbol{w}_{n}$, where $\boldsymbol{w}_{n}$ is the solution of the following equation

$$
\Pi^{\prime \prime}\left(\boldsymbol{u}_{n}\right)\left(\boldsymbol{v}, \boldsymbol{w}_{n}\right)+\Pi^{\prime}\left(\boldsymbol{u}_{n}\right)(\boldsymbol{v})=0, \quad \text { for all } \boldsymbol{v} \in \mathcal{U},
$$

or, equivalently,

$$
\mathcal{I}_{\mathrm{e}}^{\prime \prime}\left(\boldsymbol{u}_{n}\right)\left(\boldsymbol{v}, \boldsymbol{w}_{n}\right)+\mathcal{I}_{\mathrm{s}}^{\prime \prime}\left(\boldsymbol{u}_{n}\right)\left(\boldsymbol{v}, \boldsymbol{w}_{n}\right)+\mathcal{I}_{\mathrm{e}}^{\prime}\left(\boldsymbol{u}_{n}\right)(\boldsymbol{v})+\mathcal{I}_{\mathrm{s}}^{\prime}\left(\boldsymbol{u}_{n}\right)(\boldsymbol{v})-\mathcal{I}_{\mathrm{g}}^{\prime}\left(\boldsymbol{u}_{n}\right)(\boldsymbol{v})=0, \quad \text { for all } \boldsymbol{v} \in \mathcal{U},
$$

where double prime denotes the second derivative given in A.

The choice of the initial guess $\boldsymbol{u}_{0}$ is very important and determine the convergence (or absence of solution) of the Newton-Raphson algorithm. To choose $\boldsymbol{u}_{0}$, we will use the deformability parameter $\delta$ as a loading parameter. Indeed, let us remark that for $\delta=0$ the surface energy vanishes and the above problem is a standard boundary value problem in nonlinear elasticity. Its solution, denoted by 
$\boldsymbol{u}^{0}$, is the initial guess $\boldsymbol{u}_{0}$ for computing the displacement $\boldsymbol{u}=\boldsymbol{u}^{\Delta \delta}$ associated to the deformability parameter $\delta=\Delta \delta$. The displacement field $\boldsymbol{u}=\boldsymbol{u}^{(k+1) \Delta \delta}$, is then calculated step by step, by iterating the Newton-Raphson algorithm starting from $\boldsymbol{u}_{0}=\boldsymbol{u}^{k \Delta \delta}$ and increasing the deformability parameter $\delta$.

The variational formulation is written for any system of two isotropic nonlinear elastic solids $\mathcal{S}_{1}, \mathcal{S}_{2}$, but it can be easily generalised to more than two anisotropic nonlinear elastic solids. The incompressibility constraint can be taken into account by considering the constitutive law (15) rather then (12). In the following, two applications are considered: a soft drop at rest on an harder substrate (section 4) and the dual case of a stiff sphere at rest on a softer substrate (section 5). Since there is a wide interest for elastic solids which preserve their volumes, $\mathcal{S}_{1}$ and $\mathcal{S}_{2}$ will be considered incompressible. In the numerical computations, standard numerical technics (penalization, augmented Lagrangian) could be used to handle the incompressibility constraint (see for instance [Le Tallec, 1994]).

\section{A softer drop on a stiffer substrate}

Let us consider one incompressible soft elastic domain (gel-like or fluid-like) which initially is a spherical drop $\mathcal{S}_{2}$ with radius $R_{0}=\tilde{R}_{0} / L_{\mathrm{c}}$ deposited on a stiffer elastic substrate $\mathcal{S}_{1}$. Assuming axisymmetry, the substrate $\mathcal{S}_{1}$ is thought as a thick disc of depth $H=\tilde{H} / L_{\mathrm{c}}$ and radius $L=\tilde{L} / L_{\mathrm{c}}$. The origin of the polar coordinate system is in the centre of the substrate upper face (see the Fig. 1 for a representative scheme).

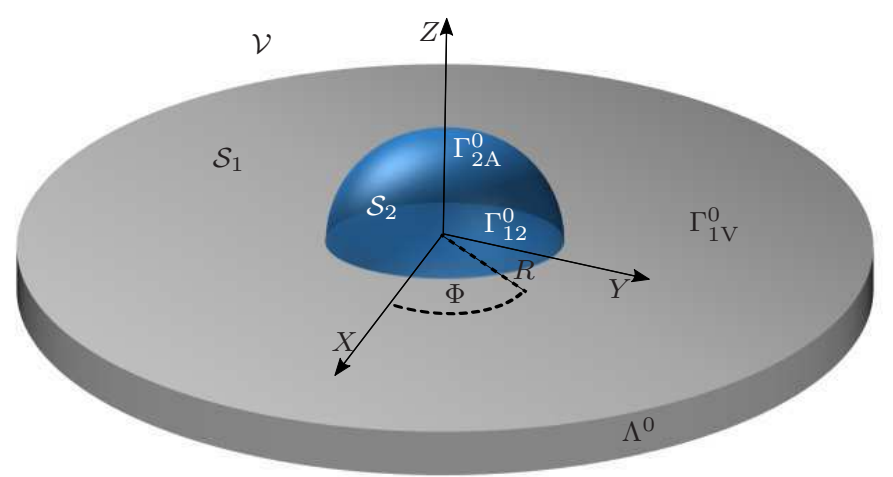

Figure 1: A soft drop $\mathcal{S}_{2}$ at rest on the elastic substrate $\mathcal{S}_{1}$ in the presence of the vapour $\mathcal{V}$. The figure shows a possible reference configuration.

\subsection{Initial configuration}

Since the uniqueness is missing the choice of the reference configuration is an important step. In the present analysis, the reference state is chosen to be the equilibrium configuration which minimizes the surface energy only, which fixes the contact line and no sliding on $\Gamma_{12}$ and $\Gamma_{1 \mathrm{~V}}$ is permitted in the analysis (pinned contact line). When the sphere $\mathcal{S}_{2}$ is assumed very soft compared to the substrate $\mathcal{S}_{1}$, this is a reasonable choice and it corresponds to $\delta=0$ by taking $\Sigma_{c}=\tilde{\mu}_{1} \rightarrow \infty$. When $\delta$ increases, the elastic effects come into play and the new equilibrium configuration will correspond to a stationary point of the total elastocapillary energy where the balance now accounts for both the elastic and surface energies (and of course the gravitationnal energy also when $\eta \neq 0$ ).

The initial configuration is computed by minimizing the surface energy with the volume conservation constraint ([White, 2003]). Basically, when no elastic contribution is considered, the equilibrium of the system is trivially the one for which $\mathcal{S}_{2}$ is a spherical cap shape at rest on the (assumed rigid) substrate. The reference $\mathcal{S}_{2}$ cap shape is defined by its radius $R$ and by the contact angle $\theta$ (see Figure 2). From simple geometrical considerations and the volume $V=4 / 3 \pi R_{0}^{3}$ conservation we get that $R=R(\theta)$,

$$
R(\theta)=\left(\frac{4 R_{0}^{3}}{(1+\cos \theta)^{2}(2-\cos \theta)}\right)^{1 / 3} .
$$




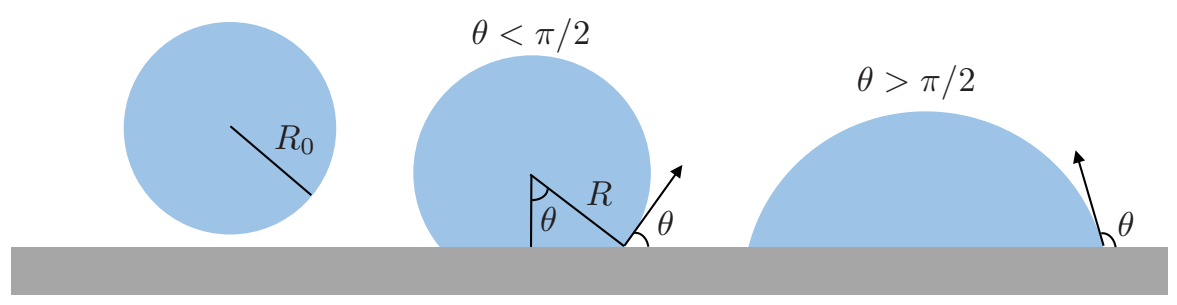

Figure 2: Initial configuration: a spherical drop of radius $R_{0}$ (left) deposited on a rigid substrate at two contact angles $\theta$ (right).

The surface energy $\mathcal{I}_{\mathrm{s}}=\gamma_{12}\left|\Gamma_{12}\right|+\gamma_{2 \mathrm{~V}}\left|\Gamma_{2 \mathrm{~V}}\right|+\gamma_{1 \mathrm{~V}}\left|\Gamma_{1 \mathrm{~V}}\right|$ depends on the surface areas $\left|\Gamma_{12}\right|=\pi(R(\theta) \sin \theta)^{2}$, $\left|\Gamma_{2 \mathrm{~V}}\right|=2 \pi R^{2}(\theta)(1+\cos \theta)$ and $\left|\Gamma_{1 \mathrm{~V}}\right|=\pi\left(L^{2}-(R(\theta) \sin \theta)^{2}\right)$ and therefore it is a function of the contact angle $\theta$ :

$$
\mathcal{I}_{\mathrm{s}}=\mathcal{I}_{\mathrm{s}}(\theta)=\pi\left(\gamma_{12}-\gamma_{1 \mathrm{~V}}\right)(R(\theta) \sin (\theta))^{2}+2 \pi \gamma_{2 \mathrm{~V}}(1+\cos \theta) R^{2}(\theta)+\pi \gamma_{1 \mathrm{~V}} L^{2}
$$

The contact angle of the initial configuration $\theta$, obtained by minimizing $\mathcal{I}_{\mathrm{s}}$ (i.e. the first variation with respect to $\theta$ vanishes) is

$$
\theta=\arccos \left(\frac{\gamma_{12}-\gamma_{1 \mathrm{~V}}}{\gamma_{2 \mathrm{~V}}}\right)
$$

The relationship (28) is refereed to as Young's equation in classical wetting theory ([Lester, 1961]) and ensures the balance of the horizontal projection of the three surface tensions at the TL.

\subsection{Boundary conditions and parameters setting}

The imposed displacement on $\Lambda^{0}$ assumes perfect adhesion and no sliding conditions-type for the bottom and lateral boundary respectively, i.e

$$
\boldsymbol{u}_{\mid Z=-H}=\mathbf{0}, \quad u_{r \mid R=L}=0 .
$$

The following parameters are chosen to reproduce typical physical parameters encountered in elastowetting experiments

$$
\gamma_{\mathrm{c}}=0.01 \mathrm{~N} / \mathrm{m}, \quad \Sigma_{\mathrm{c}}=1000 \mathrm{~N} / \mathrm{m}^{2}, \quad L_{\mathrm{c}}=0.001 \mathrm{~m}, \quad \rho_{\mathrm{c}}=1000 \mathrm{Kg} / \mathrm{m}^{3}, \quad g_{c}=10 \mathrm{~m} / \mathrm{s}^{2} .
$$

The surface parameters (surface tensions) and the geometrical parameters are chosen to be $\gamma_{12}=4.5$, $\gamma_{1 \mathrm{~V}}=1.9, \gamma_{2 \mathrm{~V}}=6.8$ and $H=0.051, L=2 R(\theta)+H, \operatorname{Vol}\left(\mathcal{S}_{2}\right)=5$. This choice of the parameters together with Young's law (28), are sufficient to uniquely define the reference domain $\mathcal{D}^{0}$ as the starting equilibrium configuration (undeformed configuration). Simulations have been carried for Mooney-Rivlin strain energy density with several choices of the parameter $f$ (see B), and since no remarkable differences being observed all results are presented in the following for the Neo-Hookean potential $(f=1 / 2)$. Finally, the computation results will be always displayed for a $2 \mathrm{D}$ half domain $\mathcal{D}$ and the blue regions will denote a softer solid while the grey region will denote the stiffer one (see coloured figures).

\subsection{Multiscale domain discretization}

In order to capture the effects near the TL on different length scales $L_{\mathrm{c}}, l_{\mathrm{s} 1}, l_{\mathrm{s} 2}$, we used a multiscale discretization of the undeformed domain $\mathcal{D}^{0}$. To unify geometric and physical length scales we choose an appropriate $a d$ hoc three levels discretization by adapting the mesh size $h$ in each region. Since the higher stresses and distortions occur next to the TL, the domain will be discretized here with a finest mesh $h_{\mathrm{s}}$, while meshes of medium size $h_{\mathrm{m}}$ cover the area along the interfaces $\Gamma$, and finally, larger meshes size $h_{1}$ are in the rest part of the domain. A mesh is then described according to the choice of $h_{\mathrm{s}}$, and of the two ratios $C_{\mathrm{ms}}$ and $C_{\mathrm{ls}}$, given by

$$
h_{\mathrm{m}}=C_{\mathrm{ms}} h_{\mathrm{s}}, \quad h_{\mathrm{l}}=C_{\mathrm{ls}} h_{\mathrm{s}} .
$$


For $C_{\mathrm{ms}} \approx 10$ and $C_{\mathrm{ls}} \approx 10^{2}$, the mesh plotted in Fig. 3 accounts for a multiscale discretization able to describe the deformation at different length scales. To obtain an accuracy in the position of the TL of the order of $10^{-2} \mu \mathrm{m} / L_{c}$ the mesh size $h_{\mathrm{s}}$ in its neighborhood has to be of the order of $1 \mu \mathrm{m} / L_{c}$. With the above multi-scale mesh the number of elements is less then $10^{4}$ and the computational costs is rather low (around 10 minutes on a laptop).

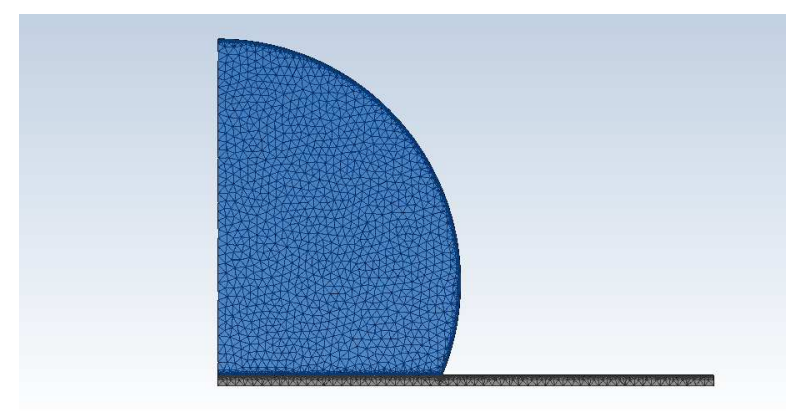

(a)

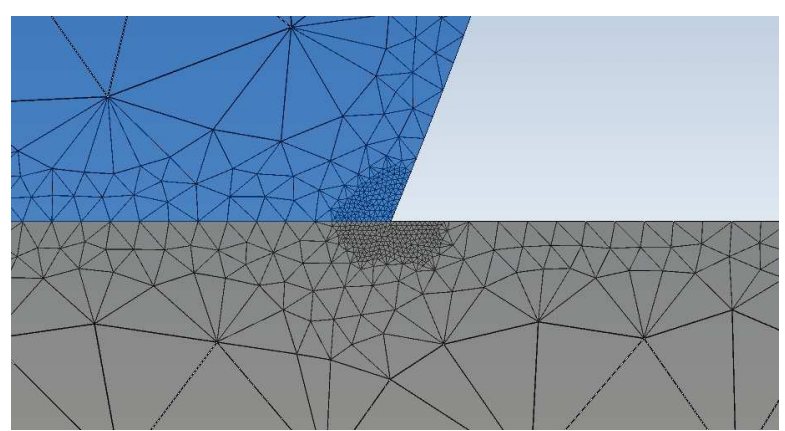

(b)

Figure 3: (a) The mesh discretization of the domain $\mathcal{D}^{0}$ following a three-level mesh size as in (31). (b) Zoom in near the TL.

\subsection{TL's position accuracy}

Several simulations have been carried in order to test the convergence of the FEM computations and the sensitivity of the TL position on the mesh size $h_{s}$. The elastic constants have been chosen to be $\mu_{1}=6, \mu_{2}=6 \times 10^{-2}$ to keep the 'softness's ratio' to be 100 (see next subsection). Keeping $C_{\mathrm{ms}}=6$ and $C_{\mathrm{ls}}=42$ fixed, five simulations have been carried for $h_{\mathrm{s}}=0.8,1,2,3$ and $4 \mu \mathrm{m} / L_{\mathrm{c}}$. As seen in Figures $4 \mathrm{a}$ and $4 \mathrm{~b}$ the computed deformed profiles of the TL are all in agreement and there are no important differences (the deformations are overlapped) for $h_{\mathrm{s}}$ less than $3 \mu \mathrm{m} / L_{\mathrm{c}}$ at a $\mu \mathrm{m}$ scale. In Fig. 5, the coordinates of the TL are reported against the sizes $h_{\mathrm{s}}$. We see clearly that for $h_{\mathrm{s}}$ less than $2 \mu \mathrm{m} / L_{\mathrm{c}}$ we get a very good accuracy (of order of $10^{-2} \mu \mathrm{m}$ ).

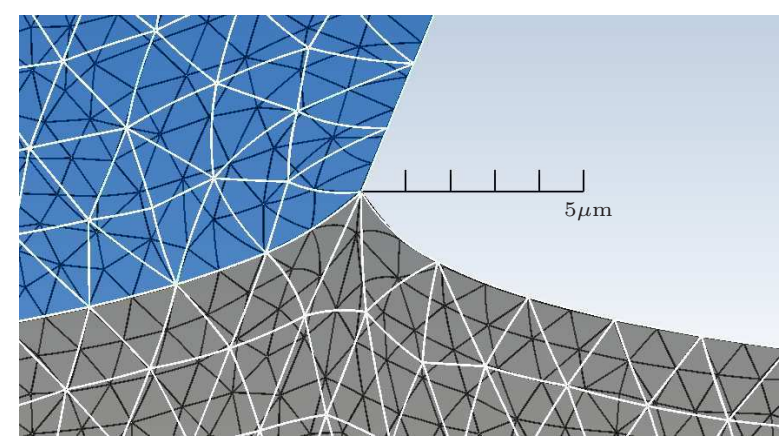

(a)

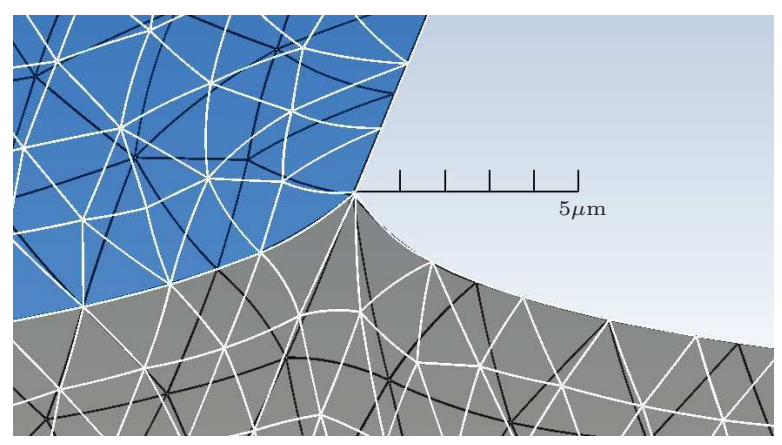

(b)

Figure 4: Comparison between the deformations near the TL for different mesh sizes $h_{\mathrm{s}}$. (a) $h_{\mathrm{s}}=1 \mu \mathrm{m} / L_{\mathrm{c}}$ (coloured regions with black wireframe) against $h_{\mathrm{s}}=2 \mu \mathrm{m} / L_{\mathrm{c}}$ (white wireframe). (b) $h_{\mathrm{s}}=2 \mu \mathrm{m} / L_{\mathrm{c}}$ (white wireframe) against $h_{\mathrm{s}}=3 \mu \mathrm{m} / L_{\mathrm{c}}$ (coloured regions with black wireframe).

\subsection{Drop 'fluidity' and gravity effects}

As mentioned previously, the problem considered in this paper, involving two solids, could also model a fluid in contact with a solid since in a fluid at rest the stress is purely hydrostatic. To simulate a fluid, we reduce the shear stress responses in the solid-like model to get a fluid-like behavior. That means that for small ratio $\mu_{2} / \mu_{1}$ the 'solid' $\mathcal{S}_{2}$ will exhibit a liquid-like behavior. We therefore conducted several simulations for different $\mu_{2} / \mu_{1}$, while $\mu_{1}$ is kept fixed. In Figure $6 \mathrm{a}$ we have compared the 


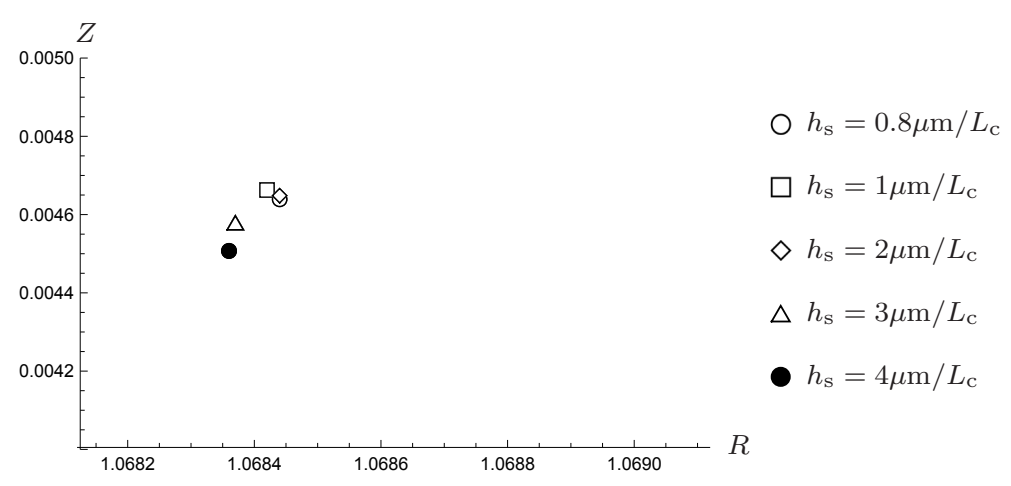

Figure 5: The TL position in a $L_{c}$-scale $(\mathrm{mm})$ for different mesh sizes $h_{\mathrm{s}}=0.8,1,2,3$ and $4 \mu \mathrm{m} / L_{\mathrm{c}}$.

deformation near the TL for $\mu_{2} / \mu_{1}=0.1$ and for $\mu_{2} / \mu_{1}=0.02$, while in Figure $6 \mathrm{~b}$ for $\mu_{2} / \mu_{1}=0.01$ and for $\mu_{2} / \mu_{1}=0.005$. We see that for $\mu_{2} / \mu_{1}$ less than 0.02 the deformation is not influenced by the shear modulus $\mu_{2}$ of the 'fluid' $\mathcal{S}_{2}$ and we conclude that for $\mu_{2} / \mu_{1}=10^{-2}$ the TL interaction between a fluid and a solid could be modeled within the framework of two solids in interaction.

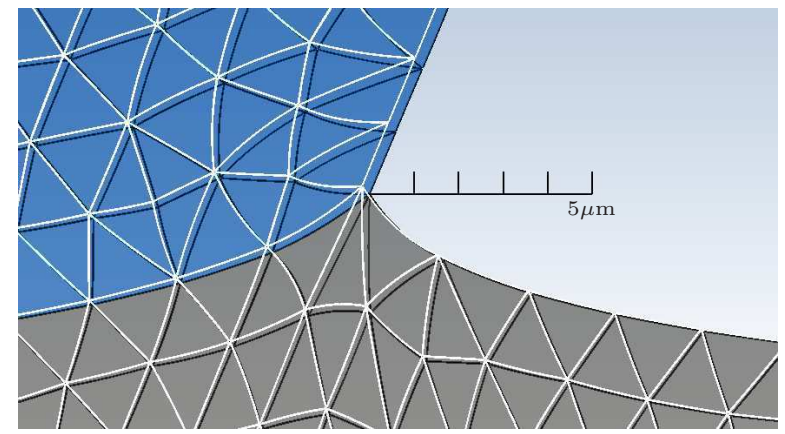

(a)

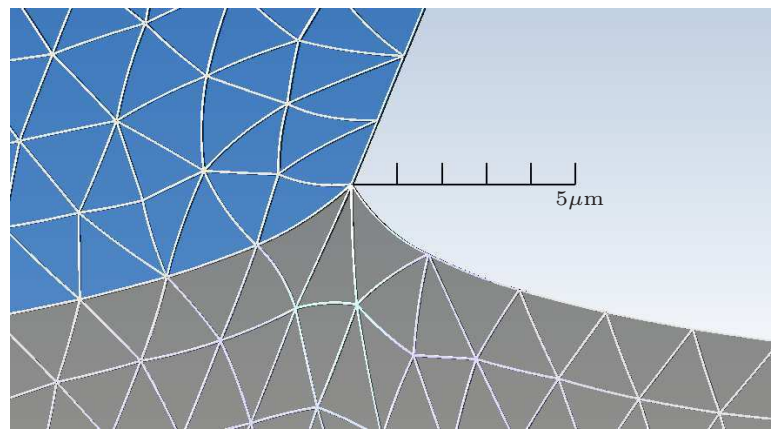

(b)

Figure 6: Comparison between the deformations near the TL for different $\mu_{2} / \mu_{1}$. (a) $\mu_{2} / \mu_{1}=0.1$ (coloured regions with black wireframe) against $\mu_{2} / \mu_{1}=0.02$ (white wireframe). (b) $\mu_{2} / \mu_{1}=0.01$ (coloured regions with black wireframe) against $\mu_{2} / \mu_{1}=0.005$ (white wireframe).

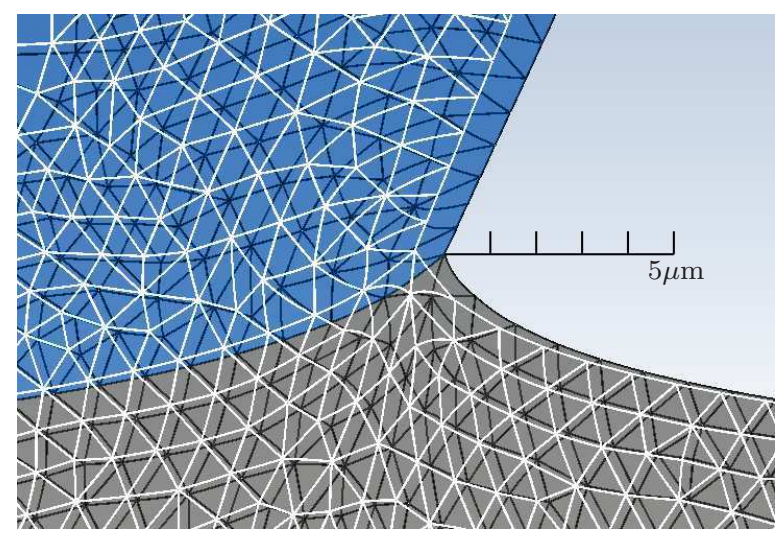

Figure 7: Comparison between the computed deformation near the TL with no gravity $(\eta=0$, white wireframe) and with gravity $\left(\eta=9.81 \times 10^{-3}\right.$, coloured mesh).

Even though gravity effects are often neglected, they can be important, even for very small drops, for the evaluation of some physical and geometrical quantities close to the TL (for example the slopes of the interfaces). In Fig. 7, we have plotted the comparison of the computed deformation near the TL with and without gravity. Clearly, near the TL at a $\mu \mathrm{m}$ scale, the predictions when gravity is not considered can lead to some substantial differences. Indeed, here, when gravity is considered, i.e. $\delta / \eta \sim 1$, the macroscopic shape of the drop changes significantly (it is not a spherical cap), and the $\mathcal{S}_{2}$ 
contact angle (or by analogy the liquid contact angle) is larger as expected. In particular, in order to quantify the change in the geometry near the TL due to the gravity, both the euclidean distances $\Delta \mathrm{TL}$ between the contact lines according if gravity is or is not considered, and amplitude of angles have been investigated. While former are mostly influenced by elasticity, the differences in the angles tend to reduce with the increasing of $\gamma_{2 \mathrm{~V}}$, (in fact increasing the latter means also increasing $\theta$ to $90^{\circ}$ where $\gamma_{2 \mathrm{~V}}$ pulls vertically). A study case is presented in the Figure 8 where several paremeters have been now replaced by $\gamma_{12}=4.0, \gamma_{1 \mathrm{~V}}=2.1, H=0.100, \mu_{1}=6,12$ (while the ratio $\mu_{2} / \mu_{1}=10^{-2}$ is kept constant) and the surface tension $\gamma_{2 \mathrm{~V}}$ tuned in the range [3,7]. The plot in Fig. 8a shows $\Delta$ TL against $\gamma_{2 \mathrm{~V}}$ for both case $\mu_{1}=6$ (black predictions) and $\mu_{1}=12$ (red predictions), while Fig. $8 \mathrm{~b}$ shows in the case $\gamma_{2 \mathrm{~V}}=3$ and $\mu_{1}=6$ the difference in the magnitude of the computed angles when gravity is considered (transparent wireframe, $\eta=9.81 \times 10^{-3}$ ) and when it is instead neglected (coloured mesh, $\eta=0$ ). Note here, that the mesh choice can not influence the angles amplitude since the same discretizazion has been used for the two simulations, and the visualization being indipendent of any measurement errors emphasizes the differences, which although might be small surely can not be discarded a priori.

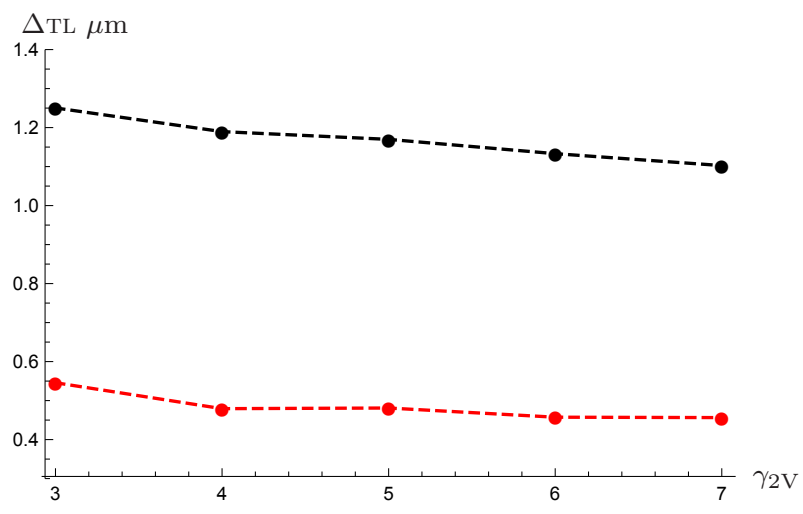

(a)

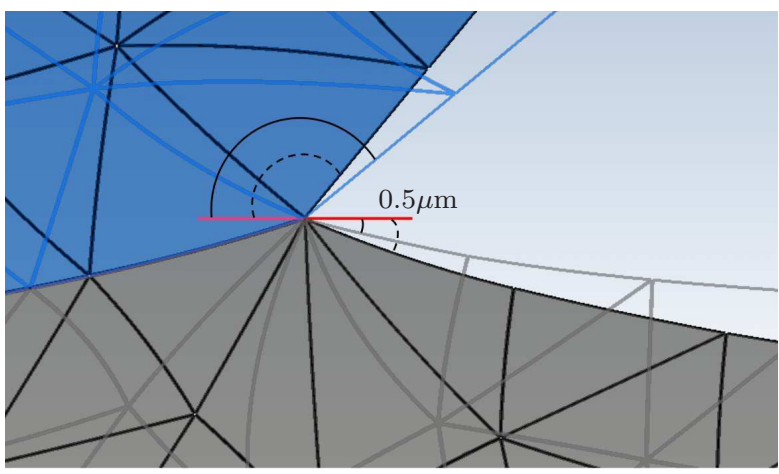

(b)

Figure 8: (a) Euclidean distance $\Delta$ TL between the computed TL which includes gravity and the TL which neglects it for both cases $\mu_{1}=6$ (black predictions) and $\mu_{2}=12$ (red predictions) against $\gamma_{2 \mathrm{~V}}$. (b) Overlapping of two images (transparent mesh for the case $\eta=9.81 \times 10^{-3} \mathrm{~m}$, and coloured mesh with a black wireframe mesh when gravity is instead neglected, $\eta=0$ ) in their TL after the latter has been rotated of $3^{\circ}$ (anticlockwise) with respect to the axis passing for its TL such that the interfaces $\Gamma_{12}$ were perfectly overlapped. The solid black circular lines sketched in the figures help to show the amplitude of the angles for the case which includes gravity while the black dashed circular lines shows the amplitude of the angles when gravity is not counted.

\subsection{Comparison between the nonlinear computations and the analytical solution of the linear elasticity}

Within the framework of linear elasticity, one may derive an analytical expression of the boundary $z=z_{1}(r)$ of the solid $\mathcal{S}_{1}$ interacting with a fluid for uniform surface tension (i.e. $\gamma_{1 \mathrm{~V}}=\gamma_{12}$ ). The problem is formulated in terms of a displacement potential function and accounts for finite thickness effects. The obtained 'analytical' solution is found in Fourier-Hankel space and must nonetheless be inverted numerically (see D for details). Of course, the linear approximation cannot be used to model situations where the tip of the ridge is bent, for which the boundary of the solid $\mathcal{S}_{1}$ cannot be written as a $z=z_{1}(r)$ function.

We compare here the nonlinear numerical computation of the deformed configuration with the analytical expression of $z=z_{1}(r)$ of $\mathcal{S}_{1}$ 's boundary obtained in linear elasticity. To do that let us consider first the symmetrical case of a substrate $\mathcal{S}_{1}$ with thickness $H=0.050$, and $\gamma_{12}=3, \gamma_{1 \mathrm{~V}}=3, \gamma_{2 \mathrm{~V}}=6.8$ and $\mu_{1}=6, \mu_{2}=\mu_{1} \times 10^{-2}$.

As we can see from Figures $9 \mathrm{a}$ and $9 \mathrm{~b}$ for symmetrical surface tensions, (i.e. $\gamma_{12}=\gamma_{1 \mathrm{~V}}$ ) and for moderate deformations $(\delta \leq 0.01)$ the linear predictions successfully recover the nonlinear theory. In 


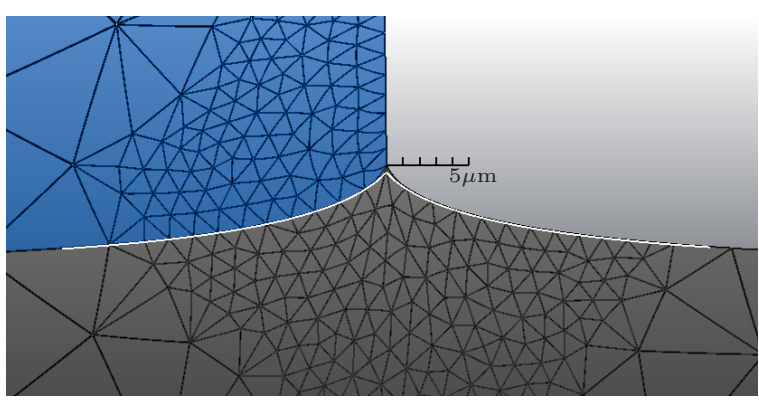

(a)

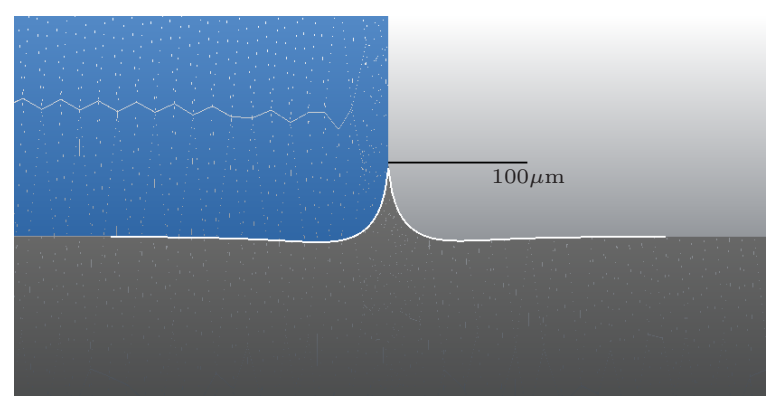

(b)

Figure 9: (a) Comparison between the nonlinear computation of the deformed configuration (coloured regions) and the linear analytical solution $z=z_{1}(r)$ (white solid line) near TL in the symmetrical case $\left(\gamma_{12}=\gamma_{1 \mathrm{~V}}\right)$ for $\delta=0.01$. (b) Same as in (a) when $z$ axis is stretched at $1: 10$.

this regime of deformability, the linear theory is still accurate to describe the deformed substrate's profile near the TL (see Fig. 9a), and excellent to describe the two dimples (see Fig. 9b) appearing on the wet and dry side respectively, far enough from the distorted area. For increasing $\delta$, as expected, the two theories diverge when approaching the TL (at a $\mu \mathrm{m}$ scale), while they are in very good agreement far enough (at a mm scale). Fig. 10a shows a comparison for $\delta=0.05$, and clearly the linear predictions are not anymore in agreement with the nonlinear ones. The Fig. 10b plots the vertical distance $\Delta$ between the nonlinear predictions of the TL and the linear predicted profile curve $^{2}$ (as specified in Fig. 10a), against the deformability parameter $\delta$. We remark that for higher values of $\delta$, the linear theory becomes less and less accurate and the difference is of order of $4 \mu \mathrm{m}$.

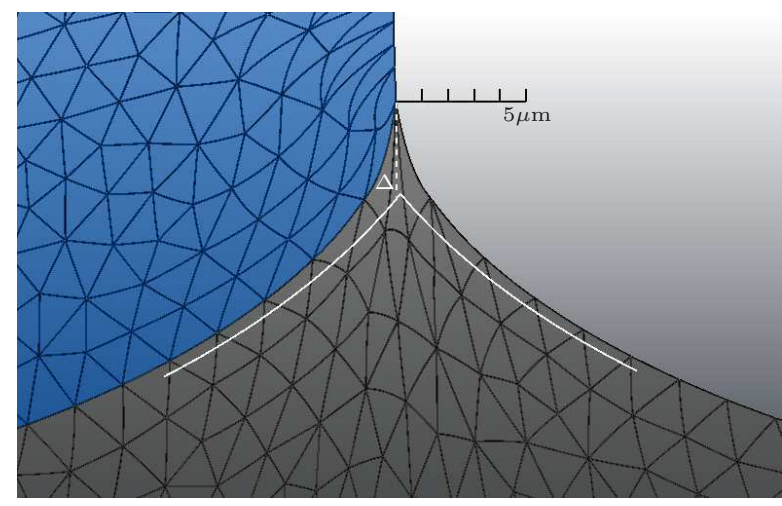

(a)

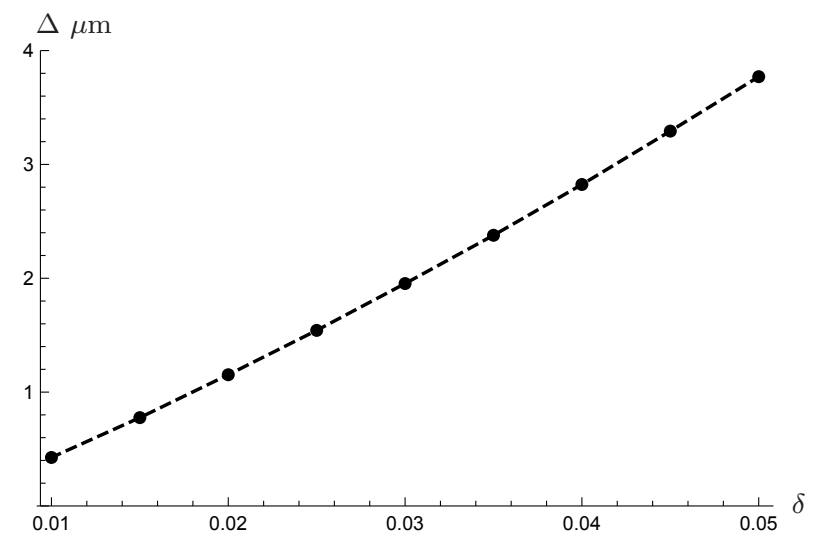

(b)

Figure 10: (a) Comparison between the nonlinear computation of the deformed configuration (coloured regions) and the linear analytical solution $z=z_{1}(r)$ (white solid line) near TL in the symmetrical case $\left(\gamma_{12}=\gamma_{1 \mathrm{~V}}\right)$ for $\delta=0.05$. (b) The vertical distance $\Delta$ (in a $\mu \mathrm{m} / L_{c}$ scale) between the linear and nonlinear predictions of the TL positions versus the deformability parameter $\delta$.

In the asymmetrical case of different solids surface tensions (i.e. $\gamma_{12}=4.5 \neq \gamma_{1 \mathrm{~V}}=1.9$ ) the linear elasticity predictions of [Bostwick et al., 2014] are not in a good agreement with the nonlinear ones even for moderate deformations $\delta=0.01$. In Fig. 11a one can see an important difference between the linear and nonlinear case for $\delta=0.01$ at the $\mu \mathrm{m}$ scale. The plot of the contour lines of the displacement gradient norm $\|\boldsymbol{\nabla} \boldsymbol{u}\|$, shows that the deformation becomes larger and larger as it approaches to the TL, and $\|\boldsymbol{\nabla} \boldsymbol{u}\|$ in some area reaches values of $130 \%$ (see Fig. 12). Under the $3 \%$ threshold, the deformations are well described accurately by the linear theory, while in the region of high values of $\|\boldsymbol{\nabla} \boldsymbol{u}\|$ the two predictions diverge. As it has already been verified experimentally (see [Park et al., 2014]) the combined effect of hydrostatic pressure and surfaces forces tend to rotate the ridge. In Fig. 11b we have plotted the nonlinear computation of the deformed configuration for a large deformability parameter $\delta=0.07$.

\footnotetext{
${ }^{2}$ The linear and nonlinear predictions of the TL are not necessarily on the same vertical line.
} 


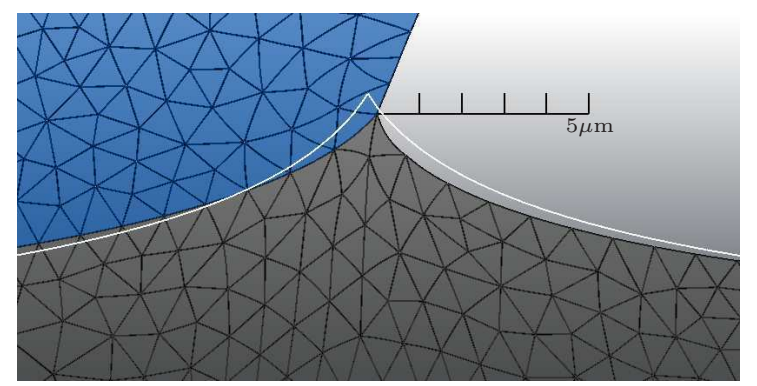

(a)

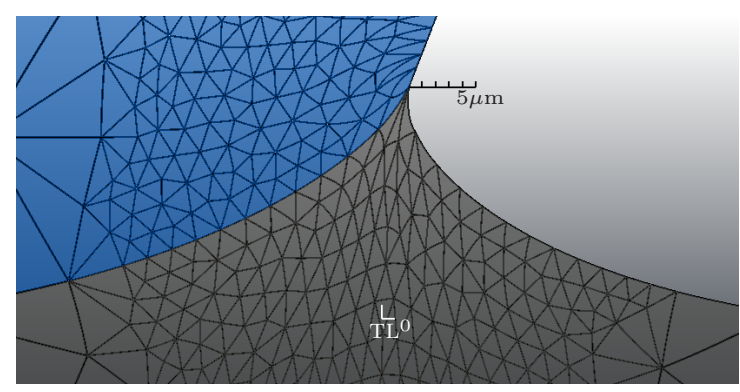

(b)

Figure 11: (a) Comparison between the nonlinear computation of the deformed configuration (coloured regions) and the linear analytical solution $z=z_{1}(r)$ (white solid line) near TL in the the asymmetrical case $\left(\gamma_{12} \neq \gamma_{1 \mathrm{~V}}\right)$ for $\delta=0.01$. (b) Nonlinear computation of the deformed configuration for $\delta=0.07$ and the cusp rotation.

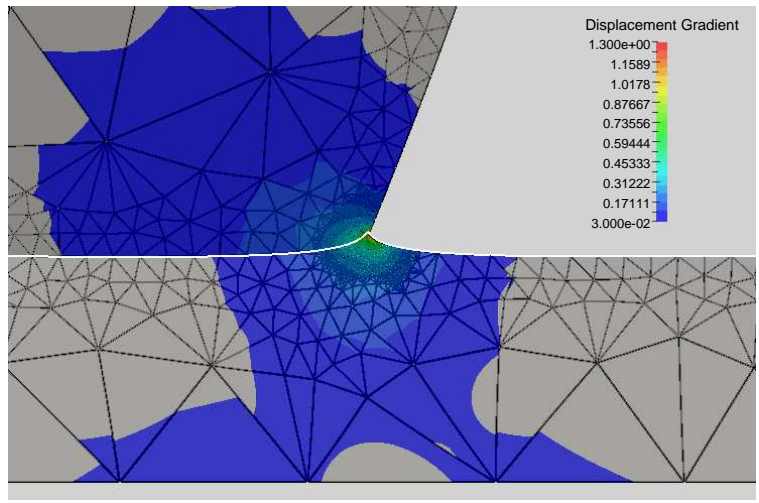

(a)

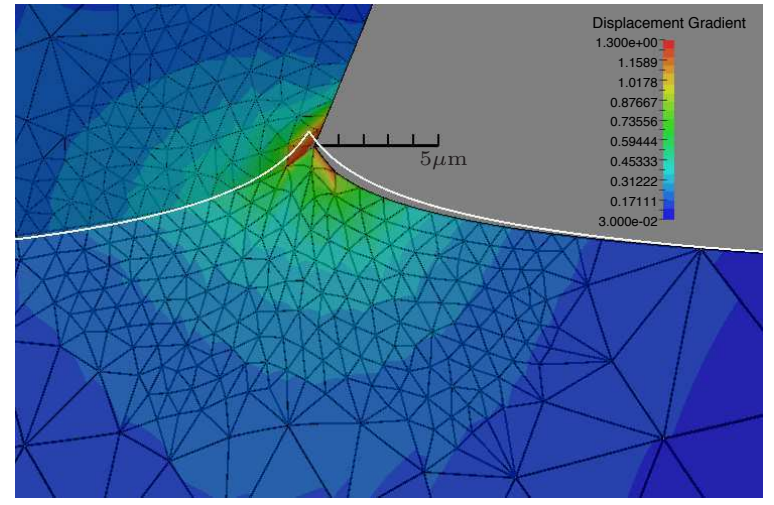

(b)

Figure 12: (a) Contour lines for the $\|\boldsymbol{\nabla} \boldsymbol{u}\| \geq 0.03$ and linear predictions (white solid curves) near the TL at a larger scale for the same settings as in Fig. 11a. (b) The same region zoomed in at a $\mu \mathrm{m}$ scale.

This clearly shows that the tip of the ridge is bent with an asymmetric tip.

\section{$5 \quad$ A stiffer drop on a softer substrate}

By contrast with the case of a soft drop at rest on a stiffer substrate, presented in the previous section 4, we present here the dual case of a stiffer elastic drop $\mathcal{S}_{2}$ deposited on a softer (gel-like) elastic substrate $\mathcal{S}_{1}$ (assumed axisymmetric with thickness $H$ and radius $L$ ). Without loosing any generality, $L$ is assumed to be a fixed radius (for instance when $\mathcal{S}_{1}$ is constrained by walls of an external circular box) and very large compared to the diameter of the drop. The solid $\mathcal{S}_{1}$ is now softer than the solid $\mathcal{S}_{2}$. The analysis will be carried by considering the following set of parameters $\gamma_{12}=4.5, \gamma_{2 \mathrm{~V}}=1.9, \gamma_{1 \mathrm{~V}}=6.8$ and $\mu_{2}=6$, $\mu_{1}=6 \times 10^{-2}$.

\subsection{Initial configuration}

Similarly to section 4.1, the initial configuration is chosen by minimizing surface energies only and thus assuming that the stiff sphere is rigid. Again, some basic geometrical considerations show that the contact angle satisfies:

$$
\theta=\arccos \left(\frac{\gamma_{12}-\gamma_{2 \mathrm{~V}}}{\gamma_{1 \mathrm{~V}}}\right) .
$$

This can also be deduced from the balance of surface tensions on the plane tangent to the sphere at the TL. The normal component of the surface tension will therefore be the main source of the deformation when the elastic energy (and gravity) is accounted for. Moreover, because of the partial immersion of 


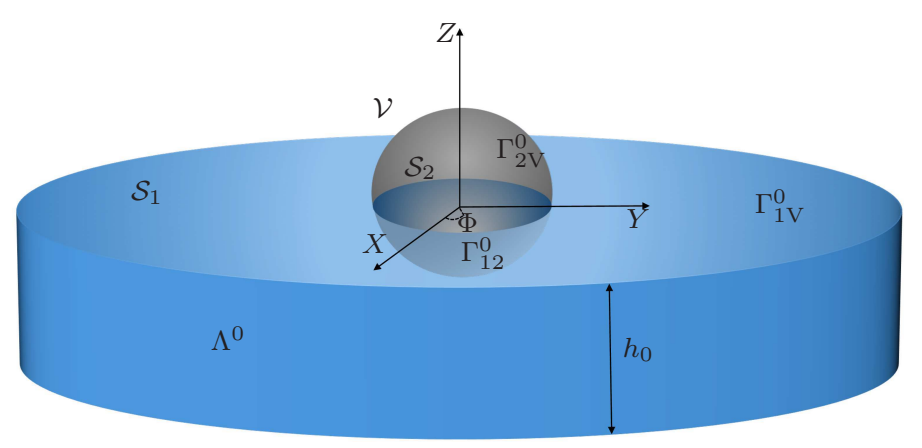

Figure 13: A stiff elastic drop $\mathcal{S}_{2}$ at rest on the softer (gel-like) elastic substrate $\mathcal{S}_{1}$ in the presence of vapour $\mathcal{V}$. The figure shows a possible reference configuration.

the drop in the substrate and the incompressibility assumption for both $\mathcal{S}_{1}$ and $\mathcal{S}_{2}$, the thickness of the substrate $H$ becomes $h$ after immersion (see Fig. 13 for a schematic visualization):

$$
h=H+\frac{R_{0}^{3}}{3 L^{2}}(1-\cos \theta)^{2}(2+\cos \theta) .
$$

The geometrical settings are now chosen to have $H=1.2, L=2 R_{0} \sin \theta+H$ and $R_{0}=1$. The domain is discretized by the multiscale approach, following the three levels mesh size proposed in section 4.3 (see Fig. 14).

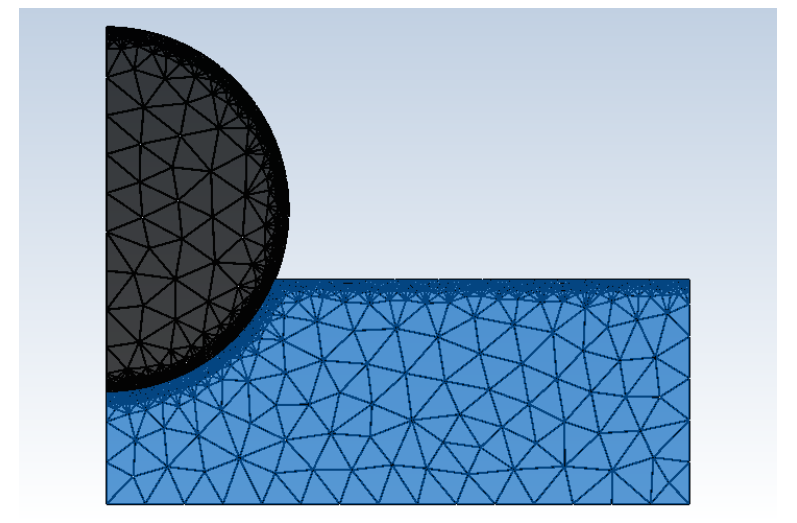

(a)

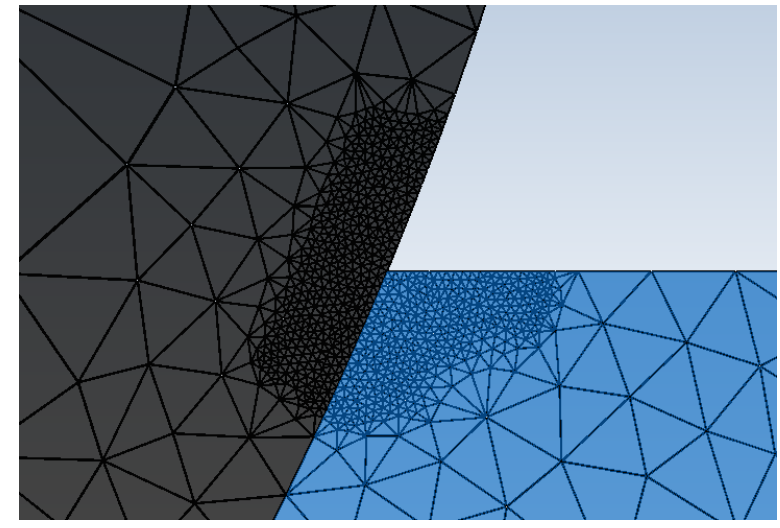

(b)

Figure 14: (a) The mesh discretization of domain $\mathcal{D}^{0}$ following a three-level mesh size as in (31). (b) Zoom near the TL.

\subsection{Deformability and gravity parameters effects}

Fig. 15a shows the comparison between the computed deformation near the TL with no gravity $(\eta=0$, unfilled wireframe) and with gravity $\eta=9.81 \times 10^{-3}$, (so that $\delta / \eta \sim 1$ ) (point filled regions) for $\delta=0.01$ with the TL marked by a red circle. The same comparison is also presented in Fig. 15b for a larger value of the deformability $\delta=0.07$. This analysis shows that even at small scales, gravity can have dramatic effects. This suggests that gravity effects must be taken into account for any theory who wishes to describe such problems.

\section{Conclusions}

We have presented in this paper a variational approach describing the equilibrium of two nonlinear elastic bodies in contact, which accounts for bulk elasticity as well as surface and gravitational energies. This work is a first step towards a generalization of linear theories, when finite deformations needs to be taken 


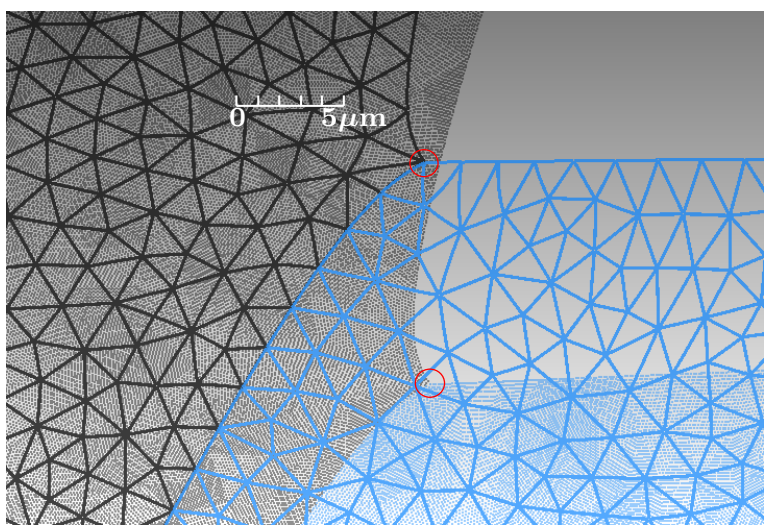

(a)

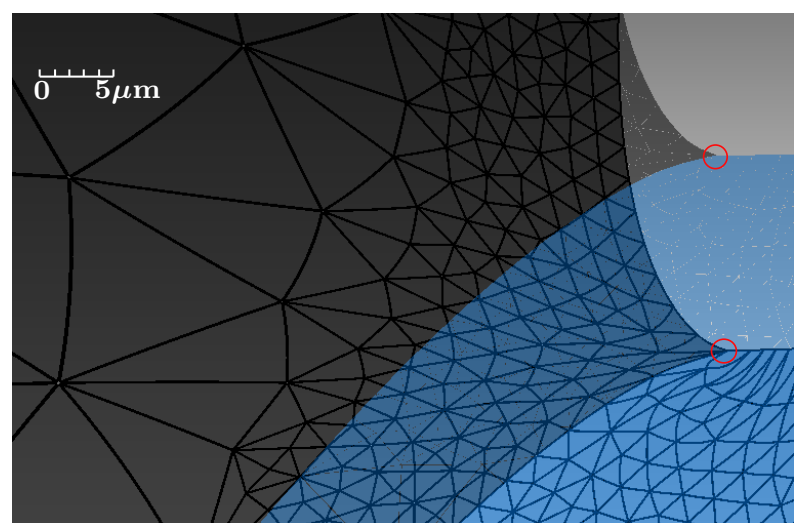

(b)

Figure 15: Comparison between the computed deformation near the TL with no gravity $(\eta=0)$ and with gravity $\left(\eta=9.81 \times 10^{-3}\right)$ for $\delta=0.01$ (a) and for $\delta=0.07$ (b). Here the 0 in the scale bar of $5 \mu \mathrm{m}$ represent the initial position of the TL in its reference configuration $\mathcal{D}^{0}$.

into account. The variational equations are derived for the most general case of two compressible solids, and the numerical Newton-Raphson scheme is adapted as a numerical tool for solving these equations. In order to tackle the inherent difficulties of the problem caused by the existence of several lengthscales, a unified deformability parameter was defined and a multiscale domain's discretization was used. We analysed the axisymmetric case of a soft drop at rest on a stiff substrate and the reciprocal problem of a stiff solid drop on a softer substrate, under the incompressibility constraint. Results are given without any restriction on the values of surface tensions. The equations were implemented in a finite element formulation coded in freeFem++.

In the first application, the simulation domain was discretized on three levels of mesh element size for which both the accuracy of the convergence and the sensitivity to the mesh size were tested. Very soft drops simulates fluid-like behavior where the static stress is purely hydrostatic. The theory derived here, which accounts for finite deformations, was also compared to the linear predictions. We have shown that, in the general case, the two theories are in agreement at low deformations but diverge for increasing deformations. In fact, even at low deformations, in the vicinity of the $\mathrm{TL}$, the displacement gradient becomes so large that any linear theory will fail. However, reasonably far from the TL, the linear predictions are rather accurate. For example, the linear theory predicts quite well the two dimples appearing in the substrate (on the wet and dry parts) and also seen experimentally. At higher deformations, and in the general case of asymmetric surface tensions, the numerical simulations reveal an asymmetry and a rotation of the tip of the ridge beneath the TL, a feature also confirmed by the experimental observations and not described by the linear theory. To model indentation applications (phenomena typically occurring at smaller scales than elastowetting problems), the second example we developed concerns the analysis and the modelling of a stiff sphere at rest on a softer substrate. In this case, we have shown that the effects of the gravity cannot be neglected since it induces significant deformations.

\section{Acknowledgements}

ANR (Agence Nationale de la Recherche) and CGI (Commisariat à l'Investissement d'Avenir) are gratefully acknowledged for their financial support through Labex SEAM (Science and Engineering for Advanced Materials and devices), ANR 11 LABX 086, ANR 11 IDEX 0502 and through the funding of the MMEMI project.

\section{A Second derivative of the total energy}

The second variation of $\mathcal{I}_{\mathrm{e}}$ is,

$$
\mathcal{I}_{\mathrm{e}}^{\prime \prime}(\boldsymbol{u})(\boldsymbol{v}, \boldsymbol{w})=\int_{\mathcal{D}^{0}}\left[\left(\frac{\partial \boldsymbol{\tau}_{K}}{\partial \mathbf{F}}(\boldsymbol{u}) \boldsymbol{\nabla} \boldsymbol{w}\right):\left(\boldsymbol{\nabla} \boldsymbol{v} \mathbf{F}^{-1}(\boldsymbol{u})\right)-\boldsymbol{\tau}_{K}(\boldsymbol{u}):\left(\boldsymbol{\nabla} \boldsymbol{v} \mathbf{F}^{-1}(\boldsymbol{u}) \boldsymbol{\nabla} \boldsymbol{w} \mathbf{F}^{-1}(\boldsymbol{u})\right)\right] \mathrm{d} \mathcal{D}_{0}
$$


and the second variation of $\mathcal{I}_{\mathrm{s}}$ is,

$$
\mathcal{I}_{\mathbf{S}}^{\prime \prime}(\boldsymbol{u})(\boldsymbol{v}, \boldsymbol{w})=\int_{\Gamma^{0}} \delta \gamma J \mathcal{F}_{1}(\boldsymbol{u}, \boldsymbol{v}, \boldsymbol{w}) \mathrm{d} \Gamma^{0}+\int_{\Gamma^{0}} \delta \gamma \mathcal{F}_{2}(\boldsymbol{u}, \boldsymbol{v}, \boldsymbol{w}) \mathrm{d} \Gamma^{0}
$$

where

$$
\begin{aligned}
\mathcal{F}_{1}(\boldsymbol{u}, \boldsymbol{v}, \boldsymbol{w})=\left\|\mathbf{F}^{-\mathrm{T}} \boldsymbol{n}^{0}\right\|\left[-\left(\mathbf{F}^{-\mathrm{T}} \boldsymbol{\nabla} \boldsymbol{w} \mathbf{F}^{-\mathrm{T}}\right): \boldsymbol{\nabla} \boldsymbol{v}+\left(\mathbf{F}^{-T}: \boldsymbol{\nabla} \boldsymbol{v}\right)\left(\mathbf{F}^{-T}: \boldsymbol{\nabla} \boldsymbol{w}\right)\right] \\
-\frac{\left(\mathbf{F}^{-\mathrm{T}} \boldsymbol{\nabla} \boldsymbol{w} \mathbf{F}^{-\mathrm{T}}\right) \boldsymbol{n}^{0} \cdot \mathbf{F}^{-\mathrm{T}} \boldsymbol{n}^{0}}{\left\|\mathbf{F}^{-\mathrm{T}} \boldsymbol{n}^{0}\right\|} \mathbf{F}^{-T}: \boldsymbol{\nabla} \boldsymbol{v}
\end{aligned}
$$

and

$$
\begin{aligned}
& \mathcal{F}_{2}(\boldsymbol{u}, \boldsymbol{v}, \boldsymbol{w})=\left[\left(\mathbf{F}^{-\mathrm{T}} \boldsymbol{\nabla} \boldsymbol{w} \mathbf{F}^{-\mathrm{T}}\right)\right.\left.\boldsymbol{\nabla} \boldsymbol{v} \mathbf{F}^{-\mathrm{T}}+\mathbf{F}^{-\mathrm{T}} \boldsymbol{\nabla} \boldsymbol{v}\left(\mathbf{F}^{-\mathrm{T}} \boldsymbol{\nabla} \boldsymbol{w} \mathbf{F}^{-\mathrm{T}}\right)\right] \boldsymbol{n}^{0} \cdot \frac{\mathbf{F}^{-\mathrm{T}} \boldsymbol{n}^{0}}{\left\|\mathbf{F}^{-\mathrm{T}} \boldsymbol{n}_{0}\right\|} \\
&+\frac{\left(\mathbf{F}^{-\mathrm{T}} \boldsymbol{\nabla} \boldsymbol{v} \mathbf{F}^{-\mathrm{T}}\right) \boldsymbol{n}^{0} \cdot\left(\mathbf{F}^{-\mathrm{T}} \boldsymbol{\nabla} \boldsymbol{w} \mathbf{F}^{-\mathrm{T}}\right) \boldsymbol{n}^{0}}{\left\|\mathbf{F}^{-\mathrm{T}} \boldsymbol{n}_{0}\right\|} \\
& \quad-\left(\left(\mathbf{F}^{-\mathrm{T}} \boldsymbol{\nabla} \boldsymbol{v} \mathbf{F}^{-\mathrm{T}}\right) \boldsymbol{n}^{0} \cdot \mathbf{F}^{-\mathrm{T}} \boldsymbol{n}^{0}\right) \frac{\left(\mathbf{F}^{-\mathrm{T}} \boldsymbol{\nabla} \boldsymbol{w} \mathbf{F}^{-\mathrm{T}}\right) \boldsymbol{n}^{0} \cdot \mathbf{F}^{-\mathrm{T}} \boldsymbol{n}^{0}}{\left\|\mathbf{F}^{-\mathrm{T}} \boldsymbol{n}_{0}\right\|^{3}},
\end{aligned}
$$

where the $\boldsymbol{u}$ dependence in $\mathbf{F}$ has been omitted for more clarity, while no contribution to the second variation of the energy is given from the gravity.

\section{B Mooney-Rivlin strain energy function}

Let A, B be second order tensors. Denoting by AB the contraction, in Cartesian components, over one repeated index and by : a contraction on two repeated indices, i.e. $\mathbf{A}: \mathbf{B}=\operatorname{Tr}\left(\mathbf{A B}^{\mathrm{T}}\right)$, we recall some simple rules of tensor calculus.

$$
\frac{\partial}{\partial \mathbf{A}} \operatorname{det}(\mathbf{A})=\mathbf{A}^{-\mathrm{T}} \operatorname{det}(\mathbf{A}), \quad \frac{\partial \mathbf{A}^{-1}}{\partial \mathbf{A}}: \mathbf{B}=-\mathbf{A}^{-1} \mathbf{B} \mathbf{A}^{-1}, \quad \frac{\partial \mathbf{A}^{-\mathrm{T}}}{\partial \mathbf{A}}: \mathbf{B}=-\mathbf{A}^{-\mathrm{T}} \mathbf{B} \mathbf{A}^{-\mathrm{T}} .
$$

Let us consider the strain energy function $W_{\mathrm{e}}$ of the Mooney-Rivlin type

$$
W_{\mathrm{e}}^{M R}=\frac{1}{2}\left(\frac{1}{2}+f\right) \mu\left(I_{1}-3\right)+\frac{1}{2}\left(\frac{1}{2}-f\right) \mu\left(I_{2}-3\right),
$$

where $f$ is a dimensionless constant in the range $-1 / 2 \leq f \leq 1 / 2$ and $\mu>0$ is the shear modulus for infinitesimal deformations. Note when $f=1 / 2$, the neo-Hookean model is recovered. The Kirchhoff stress tensor reads now

$$
\boldsymbol{\tau}_{K}=-p \mathbf{I}+\mu\left(\left(\frac{1}{2}+f\right)+\left(\frac{1}{2}-f\right) I_{1}\right) \mathbf{B}-\mu\left(\frac{1}{2}-f\right) \mathbf{B}^{2}
$$

and its deviatoric part

$$
\boldsymbol{\tau}_{K}^{\mathrm{D}}=\mu\left(\left(\frac{1}{2}+f\right)+\left(\frac{1}{2}-f\right) I_{1}\right) \mathbf{B}-\mu\left(\frac{1}{2}-f\right) \mathbf{B}^{2}-\frac{\mu}{3}\left(\left(\frac{1}{2}+f\right) I_{1}+2\left(\frac{1}{2}-f\right) I_{2}\right) \mathbf{I}
$$

while

$$
\begin{aligned}
\frac{\partial \boldsymbol{\tau}_{K}}{\partial \mathbf{F}} \mathbf{A}=\mu\left(\left(\frac{1}{2}-f\right) 2 \mathbf{F}: \mathbf{A}\right) \mathbf{B}+\mu\left(\left(\frac{1}{2}+f\right)+\left(\frac{1}{2}-f\right) I_{1}\right)\left(\mathbf{A} \mathbf{F}^{\mathrm{T}}+\mathbf{F A}^{\mathrm{T}}\right) \\
-\mu\left(\frac{1}{2}-f\right)\left(\left(\mathbf{A} \mathbf{F}^{\mathrm{T}}+\mathbf{F} \mathbf{A}^{\mathrm{T}}\right) \mathbf{B}+\mathbf{B}\left(\mathbf{A} \mathbf{F}^{\mathrm{T}}+\mathbf{F} \mathbf{A}^{\mathrm{T}}\right)\right)
\end{aligned}
$$




\section{The axisymmetric case}

Under the assumption of axisymmetry, reference and current configurations, $\mathcal{D}^{0}$ and $\mathcal{D}$, respectively, can therefore be described by the use of cylindrical coordinates $(R, Z, \Phi)$ and $(r, z, \varphi)$, respectively, where

$$
r=r(R, Z), \quad z=z(R, Z), \quad \varphi=\Phi(0 \leq \Phi<2 \pi),
$$

and $\boldsymbol{u}=\left(u_{r}(R, Z), u_{z}(R, Z), 0\right)$. It is also assumed

$$
\begin{array}{ll}
\mathcal{D}^{0}=\left\{(R, Z) \in \Omega^{0}, \Phi \in[0,2 \pi)\right\}, & \mathcal{D}=\{(r, z) \in \Omega, \varphi \in[0,2 \pi)\}, \\
\Gamma^{0}=\left\{(R, Z) \in \Gamma_{2}^{0}, \Phi \in[0,2 \pi)\right\}, & \Gamma=\left\{(r, z) \in \Gamma_{2}, \varphi \in[0,2 \pi)\right\}, \\
\Lambda^{0}=\left\{(R, Z) \in \Lambda_{2}^{0}, \Phi \in[0,2 \pi)\right\}, & \Lambda=\left\{(r, z) \in \Lambda_{2}, \varphi \in[0,2 \pi)\right\},
\end{array}
$$

where $\Omega^{0}$ and $\Omega$, as well as $\Gamma_{2}^{0}, \Lambda_{2}^{0}$ and $\Gamma_{2}, \Lambda_{2}$ are the 2-D domains, and 2-D boundaries, in the plane $\left\langle\boldsymbol{e}_{R}, \boldsymbol{e}_{Z}\right\rangle$ and $\left\langle\boldsymbol{e}_{r}, \boldsymbol{e}_{z}\right\rangle$, respectively (see Fig. 16).
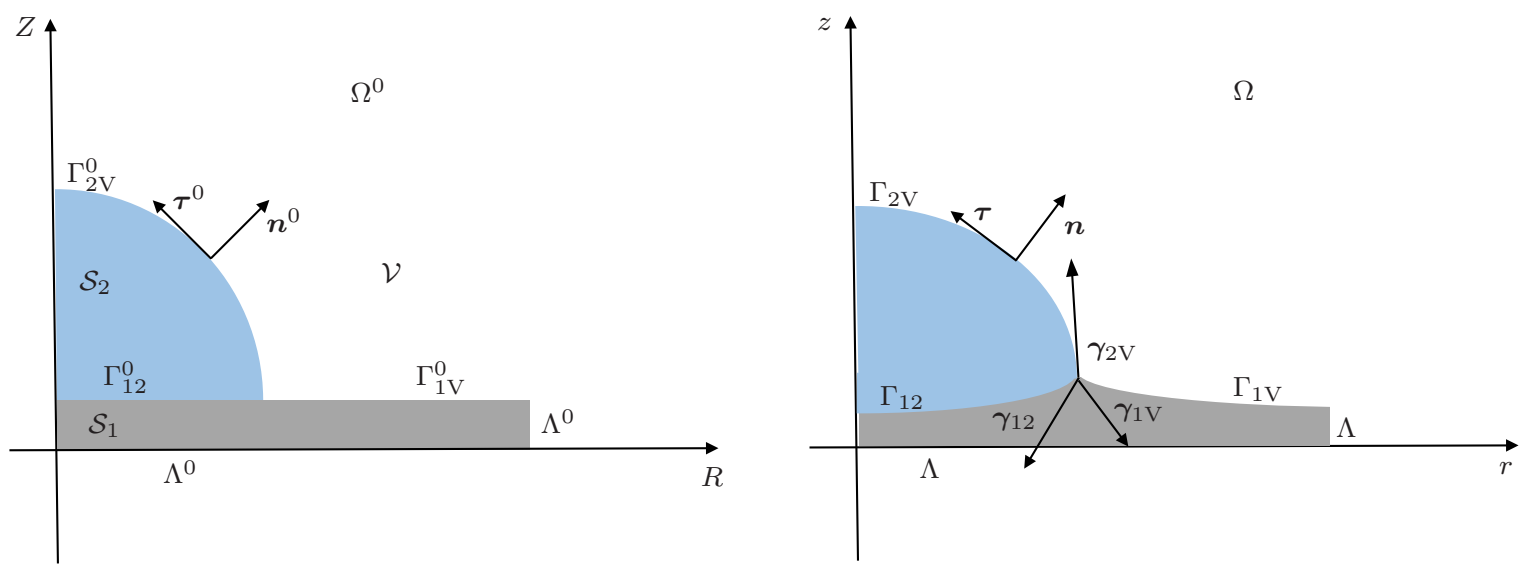

Figure 16: Half section of a 2D axisymmetric soft gel-like drop $\mathcal{S}_{2}$ on a harder substrate $\mathcal{S}_{1}$ with the presence of some vapour $\mathcal{V}$, represented in a schematic case in its reference configuration $\Omega^{0}$ (left) and in its current configuration $\Omega$ (right), no scale is implied.

The displacement gradient $\boldsymbol{\nabla} \boldsymbol{u}$ and the deformation gradient $F_{i j}=\partial x_{i} / \partial X_{j}$ associated with (43) in polar components are

$$
\boldsymbol{\nabla} \boldsymbol{u}=\left[\begin{array}{cc}
{[\boldsymbol{\nabla} \boldsymbol{u}]_{2}} & 0 \\
0 & u_{r} / R
\end{array}\right], \quad \boldsymbol{F}=\left[\begin{array}{cc}
\boldsymbol{F}_{2} & 0 \\
0 & r / R
\end{array}\right]
$$

while the left Cauchy-Green tensor $\mathbf{B}=\mathbf{F F}^{\mathrm{T}}$ and its inverse are given by

$$
\mathbf{B}=\left[\begin{array}{cc}
\mathbf{B}_{2} & 0 \\
0 & (r / R)^{2}
\end{array}\right], \quad \mathbf{B}^{-1}=\left[\begin{array}{cc}
\mathbf{B}_{2}^{-1} & 0 \\
0 & (R / r)^{2}
\end{array}\right]
$$

where

$$
\begin{gathered}
{[\boldsymbol{\nabla} \boldsymbol{u}]_{2}=\left[\begin{array}{cc}
u_{r, R} & u_{r, Z} \\
u_{z, R} & u_{z, Z}
\end{array}\right], \quad \mathbf{F}_{2}=\left[\begin{array}{cc}
r_{, R} & r_{, Z} \\
z_{, R} & z_{, Z}
\end{array}\right], \quad \mathbf{B}_{2}=\left[\begin{array}{cc}
r_{, R}^{2}+r_{, Z}^{2} & r_{R} z_{, R}+r_{Z} z_{, Z} \\
r_{R} z_{, R}+r_{Z} z_{, Z} & z_{, R}^{2}+z_{, Z}^{2}
\end{array}\right],} \\
\mathbf{B}_{2}^{-1}=\left[\begin{array}{cc}
z_{, R}^{2}+z_{, Z}^{2} & -\left(r_{R} z_{, R}+r_{Z} z_{, Z}\right) \\
-\left(r_{R} z_{, R}+r_{Z} z_{, Z}\right) & r_{, R}^{2}+r_{, Z}^{2}
\end{array}\right] /\left(r_{Z} z_{, R}-r_{, R} z_{, Z}\right)^{2},
\end{gathered}
$$

and where the, denotes the partial derivative with respect to the subscript variable. Moreover

$$
I_{3}=J_{2}^{2}\left(\frac{r}{R}\right)^{2}, \quad J_{2}=\operatorname{det} \mathbf{F}_{2} .
$$


Where defined, let $\boldsymbol{\tau}, \boldsymbol{n}$ be the unit tangent and outward normal vectors, respectively,

$$
\boldsymbol{\tau}=\left(\boldsymbol{\tau}_{2}, 0\right), \quad \boldsymbol{n}=\left(\boldsymbol{n}_{2}, 0\right),
$$

where

$$
\boldsymbol{\tau}_{2}=\left(\tau_{r}, \tau_{z}\right), \quad \boldsymbol{n}_{2}=\left(n_{r}, n_{z}\right),
$$

are the respective two-dimensional unit tangent and outward normal vectors. Since

$$
J\left\|\mathbf{F}^{-\mathrm{T}} \boldsymbol{n}^{0}\right\|=J_{2} \frac{r}{R}\left\|\mathbf{F}_{2}^{-\mathrm{T}} \boldsymbol{n}_{2}^{0}\right\|=\frac{r}{R}\left\|\mathbf{F}_{2} \boldsymbol{\tau}_{2}^{0}\right\|,
$$

where the last equality is obtained from a two-dimensional version of Nanson's formula (see [Steigmann and Ogden, the superficial term in (10) can therefore be rewritten as

$$
\mathcal{I}_{\mathrm{s}}=\int_{\Gamma^{0}} \delta \gamma J\left\|\mathbf{F}^{-\mathrm{T}} \boldsymbol{n}^{0}\right\| \mathrm{d} \Gamma^{0}=2 \pi \delta \int_{\Gamma_{2}^{0}} \gamma r\left\|\mathbf{F}_{2} \boldsymbol{\tau}_{2}^{0}\right\| \mathrm{d} R \mathrm{~d} Z
$$

where the integration over $\Phi$ has been computed. From definition (18), the first variation of $\mathcal{I}_{\mathrm{S}}(\boldsymbol{u})$ is easily derived as

$$
\mathcal{I}_{\mathbf{s}}^{\prime}(\boldsymbol{u})(\boldsymbol{v})=2 \pi \delta \int_{\Gamma_{2}^{0}} \gamma\left(R+u_{r}\right) \frac{\mathbf{F}_{2} \boldsymbol{\tau}_{2}^{0}}{\left\|\mathbf{F}_{2} \boldsymbol{\tau}_{2}^{0}\right\|} \cdot \boldsymbol{\nabla} \boldsymbol{v} \tau_{2}^{0} \mathrm{~d} R \mathrm{~d} Z+2 \pi \delta \int_{\Gamma_{2}^{0}} \gamma v_{r}\left\|\mathbf{F}_{2}^{0} \tau_{2}^{0}\right\| \mathrm{d} R \mathrm{~d} Z
$$

for every $\boldsymbol{v}=\left(v_{r}(R, Z), v_{z}(R, Z), 0\right)$ in $\mathcal{U}$. The first variation of $\mathcal{I}_{\mathrm{e}}$ reads

$$
\mathcal{I}_{\mathrm{e}}^{\prime}(\boldsymbol{u})(\boldsymbol{v})=2 \pi \int_{\Omega^{0}} \mathbf{P}(\boldsymbol{u}): \nabla \boldsymbol{v} R \mathrm{~d} R \mathrm{~d} Z=2 \pi \int_{\Omega^{0}} \boldsymbol{\tau}_{K}(\boldsymbol{u}): \nabla \boldsymbol{v} \mathbf{F}^{-1}(\boldsymbol{u}) R \mathrm{~d} R \mathrm{~d} Z
$$

while the first variation for the gravity energy term as

$$
\mathcal{I}_{\mathrm{g}}^{\prime}(\boldsymbol{u})(\boldsymbol{v})=2 \pi \int_{\Omega^{0}} \eta \boldsymbol{g} \cdot \boldsymbol{v} R \mathrm{~d} R \mathrm{~d} Z
$$

In order to apply the Newton-Raphson procedure (24), the second variation of $\mathcal{I}_{\mathrm{e}}$ is,

$$
\begin{aligned}
\mathcal{I}_{\mathrm{e}}^{\prime \prime}(\boldsymbol{u})(\boldsymbol{v}, \boldsymbol{w}) & =2 \pi \int_{\Omega^{0}}\left(\frac{\partial \mathbf{P}}{\partial \mathbf{F}}(\boldsymbol{u}) \boldsymbol{\nabla} \boldsymbol{w}\right): \boldsymbol{\nabla} \boldsymbol{v} R \mathrm{~d} R \mathrm{~d} Z \\
= & 2 \pi \int_{\Omega^{0}}\left[\left(\frac{\partial \boldsymbol{\tau}_{K}}{\partial \mathbf{F}}(\boldsymbol{u}) \boldsymbol{\nabla} \boldsymbol{w}\right):\left(\boldsymbol{\nabla} \boldsymbol{v} \mathbf{F}^{-1}(\boldsymbol{u})\right)-\boldsymbol{\tau}_{K}(\boldsymbol{u}):\left(\boldsymbol{\nabla} \boldsymbol{v} \mathbf{F}^{-1}(\boldsymbol{u}) \boldsymbol{\nabla} \boldsymbol{w} \mathbf{F}^{-1}(\boldsymbol{u})\right)\right] R \mathrm{~d} R \mathrm{~d} Z
\end{aligned}
$$

and the second variation of $\mathcal{I}_{\mathrm{S}}$ is,

$$
\begin{aligned}
\mathcal{I}_{\mathbf{s}}^{\prime \prime}(\boldsymbol{u})(\boldsymbol{v}, \boldsymbol{w}) & =2 \pi \delta \int_{\Gamma_{2}^{0}} \frac{\gamma\left(R+u_{r}\right)}{\left\|\mathbf{F}_{2}(\boldsymbol{u}) \boldsymbol{\tau}_{2}^{0}\right\|^{3}}\left[\left\|\mathbf{F}_{2}(\boldsymbol{u}) \boldsymbol{\tau}_{2}^{0}\right\|^{2} \boldsymbol{\nabla} \boldsymbol{w}_{n} \boldsymbol{\tau}_{2}^{0}-\left(\boldsymbol{\nabla} \boldsymbol{w} \tau_{2}^{0} \cdot \mathbf{F}_{2}(\boldsymbol{u}) \boldsymbol{\tau}^{0}\right) \mathbf{F}_{2}(\boldsymbol{u}) \boldsymbol{\tau}_{2}^{0}\right] \cdot \boldsymbol{\nabla} \boldsymbol{v} \boldsymbol{\tau}_{2}^{0} R \mathrm{~d} R \mathrm{~d} Z \\
& +2 \pi \delta \int_{\Gamma_{2}^{0}} \gamma u_{r} \frac{\mathbf{F}_{2}(\boldsymbol{u}) \boldsymbol{\tau}_{2}^{0}}{\left\|\mathbf{F}_{2}(\boldsymbol{u}) \boldsymbol{\tau}_{2}^{0}\right\|} \cdot \nabla \boldsymbol{v} \tau_{2}^{0} R \mathrm{~d} R \mathrm{~d} Z+2 \pi \delta \int_{\Gamma_{2}^{0}} \gamma v_{r} \frac{\mathbf{F}_{2}(\boldsymbol{u}) \boldsymbol{\tau}_{2}^{0}}{\left\|\mathbf{F}_{2}(\boldsymbol{u}) \boldsymbol{\tau}_{2}^{0}\right\|} \cdot \boldsymbol{\nabla} \boldsymbol{w} \tau_{2}^{0} R \mathrm{~d} R \mathrm{~d} Z
\end{aligned}
$$

\section{The linear elastic solution}

The deformation of the elastic solid is described by the axisymmetric displacement field $\boldsymbol{u}$,

$$
\boldsymbol{u}=u_{r}(r, z) \boldsymbol{e}_{r}+u_{z}(r, z) \boldsymbol{e}_{z},
$$

and the strain tensor $\boldsymbol{\epsilon}$ is defined as

$$
\boldsymbol{\epsilon}=\frac{1}{2}\left(\boldsymbol{\nabla} \boldsymbol{u}+(\boldsymbol{\nabla} \boldsymbol{u})^{\mathrm{T}}\right)
$$


while the linear stress stress relationships gives the stress tensor $\sigma$ as

$$
\boldsymbol{\sigma}=\frac{E}{1+\nu}\left(\boldsymbol{\epsilon}+\frac{\nu}{1-2 \nu} \operatorname{Tr}(\boldsymbol{\epsilon}) \boldsymbol{I}\right)
$$

where $E$ is the Young's modulus and $\nu$ is the Poisson ratio. The mechanical equilibrium in the bulk of the soft elastic layer is described by the Navier equations:

$$
\nabla \cdot \sigma=0
$$

or, using the displacement field $\boldsymbol{u}$ by

$$
(1-2 \nu) \triangle \boldsymbol{u}+\boldsymbol{\nabla}(\boldsymbol{\nabla} \cdot \boldsymbol{u})=\mathbf{0} .
$$

This set of equations is completed by the condition of stress continuity at the free boundary $z=h$,

$$
\boldsymbol{\sigma} \boldsymbol{n}=\boldsymbol{t}+\gamma_{s} \boldsymbol{n}(\boldsymbol{\nabla} \cdot \boldsymbol{n})
$$

where $\boldsymbol{n}$ and $\boldsymbol{t}$ are the unit normal vector to the surface and traction forces exerted at the substrate boundary, respectively. $\gamma_{s l}$ is the surface energy of the solid-liquid interface and $\gamma_{s g}$ is the surface energy of the solid-gas interface. In addition, the soft elastic layer is bounded at the bottom, i.e at $z=0$,

$$
\boldsymbol{u}(r, 0)=\mathbf{0} .
$$

Let us consider the problem of a static purely normal force $\boldsymbol{t}=F_{z}(r) \boldsymbol{e}_{z}$ applied at the free boundary of the elastic solid. The axisymmetric compressible Navier equations are simplified by introducing a potential function known as the Galerkin vector $\mathbf{G}$,

$$
\mathbf{G}=\xi(r, z) \boldsymbol{e}_{z}
$$

defined as

$$
\boldsymbol{u}=\frac{1+\nu}{E}(2(1-\nu) \triangle \mathbf{G}-\nabla(\boldsymbol{\nabla} \cdot \mathbf{G})) .
$$

Substituting (67) into (63), we obtain the familiar biharmonic equation of linear elasticity

$$
\triangle^{2} \xi=0 .
$$

In the axisymmetric geometry, it is useful to defined the Fourier-Hankel transform with respect to $\mathrm{r}$ by

$$
\hat{\xi}(s, z)=\int_{0}^{\infty} r \xi(r, z) J_{0}(s r) \mathrm{d} r
$$

and its inverse transform by

$$
\xi(r, z)=\int_{0}^{\infty} s \hat{\xi}(s, z) J_{0}(s r) \mathrm{d} s .
$$

Inserting (70) into the biharmonic equation (68), we get the simple fourth-order ordinary differential equation for $\hat{\xi}$,

$$
\frac{\mathrm{d}^{4} \hat{\xi}}{\mathrm{d} z^{4}}-2 s^{2} \frac{\mathrm{d}^{2} \hat{\xi}}{\mathrm{d} z^{2}}+s^{4} \hat{\xi}=0
$$

and the boundary condition (65) can be rewritten in term of $\hat{\xi}(s, z)$ as

$$
\frac{\mathrm{d} \hat{\xi}}{\mathrm{d} z}=0 \text { and }(1-2 \nu) \frac{\mathrm{d}^{2} \hat{\xi}}{\mathrm{d} z^{2}}-2(1-\nu) s^{2} \hat{\xi}=0
$$

The general solution of (71), subjected to the two boundary conditions in (72), is then

$$
\hat{\xi}(s, z)=C(s)\left(\cosh (s z)+\frac{s z \sinh (s z)}{2(1-2 \nu}\right)+D(s)(s z \cosh (s z)-\sinh (s z))
$$


where $C(s)$ and $D(s)$ are two functions of $s$ that need to be determined using the remaining two scalar boundary conditions (64) at the free surface $z=h$. In component forms, (64) reads

$$
\sigma_{r z}=0 \text { for } 0 \leq r \leq \infty
$$

and

$$
\sigma_{z z}-\gamma_{s} \triangle_{\|} u_{z}-F_{z}=0
$$

where $\triangle_{\|}$is the Laplacian operator along the $r$ direction only. Using the constitutive relation (61) together with definition (67) of the Galerkin vector, the first boundary condition (74) reads,

$$
(1-\nu) \frac{\partial \xi}{\partial r}+r\left(r \nu \frac{\partial^{3} \xi}{\partial r \partial z^{2}}-(1-\nu)\left(\frac{\partial^{2} \xi}{\partial r^{2}}+r \frac{\partial^{3} \xi}{\partial r^{3}}\right)\right)_{\mid z=h}=0
$$

and inserting the definition (70) of the Fourier-Hankel transform into (76) we get

$$
s^{2}(1-\nu) \hat{\xi}(s, h)+\left.\nu \frac{\partial^{2} \hat{\xi}}{\partial z^{2}}\right|_{z=h}=0 .
$$

Now inserting the solution (73) into (77) we get a relation between $C(s)$ and $D(s)$,

$$
D(s)=C(s) \frac{2(1-\nu) \cosh (h s)+h s \sinh (h s)}{2(1-2 \nu)((1-2 \nu) \sinh (h s)-h s \cosh (h s))} .
$$

We now have a single function $C(s)$ to be determined using the boundary condition (75). To find the solution we rewrite this BC using the inverse Hankel transform (70),

$$
\hat{\sigma}_{z z}(s, h)+\gamma_{s} s^{2} \hat{u}_{z}(s, h)-\hat{F}_{z}(s)=0
$$

which solution is

$$
\begin{aligned}
& C(s)=\left(4 E(2 \nu-1) \hat{F}_{z}(s)((2 \nu-1) \sinh (h s)+h s \cosh (h s))\right) / \\
& \left(s^{3}\left(E\left(2 h^{2} s^{2}+4 \nu(2 \nu-3)+5\right)+E(3-4 \nu) \cosh (2 h s)+4 \gamma_{s} h\left(\nu^{2}-1\right) s^{2}+2 \gamma_{s}\left(\nu^{2}-1\right)(4 \nu-3) s \sinh (2 h s)\right)\right)
\end{aligned}
$$

Equation (73), together with (78) and (80) is the general solution of the problem of a compressible substrate of finite thickness $h$ under an arbitrary distribution of normal forces. We are mostly interested by the deformation of the free surface of an incompressible material. Evaluating (73) at $z=h$ and in the limit $\nu \rightarrow 1 / 2$, the surface displacement field is given by

$$
u_{z}(r, h)=\int_{0}^{\infty} \frac{\hat{F}_{z}(s) J_{0}(s r)}{s \gamma_{s}\left(\frac{2 E\left(2 h^{2} s^{2}+\cosh (2 h s)+1\right)}{3 \gamma_{s}(\sinh (2 h s)-2 h s)}+s\right)} \mathrm{d} s
$$

Since for a spherical drop with radius $R$ and contact angle $\alpha$, the normal force is given by

$$
\hat{F}_{z}=\gamma \sin (\alpha)\left(R J_{0}(s R)-\frac{2}{s} J_{1}(s R)\right)
$$

where $\gamma$ is the surface energy of the liquid-gas interface, the deformation profile for the symmetrical case $\alpha=\pi / 2$ (i.e. $\gamma_{s}=\gamma_{s g}=\gamma_{s l}$ ) is finally given by

$$
u_{z}(r, h)=\frac{\gamma}{\gamma_{s}} \int_{0}^{\infty} \frac{J_{0}(s r)\left(R J_{0}(s R)-\frac{2}{s} J_{1}(s R)\right)}{s\left(\frac{2 E\left(2 h^{2} s^{2}+\cosh (2 h s)+1\right)}{3 \gamma_{s}(\sinh (2 h s)-2 h s)}+s\right)} \mathrm{d} s .
$$




\section{References}

[Ben Amar and Ciarletta, 2010] Ben Amar, M. and Ciarletta, P. (2010). Swelling instability of surfaceattached gels as a model of soft tissue growth under geometric constraints. Journal of the Mechanics and Physics of Solids, 58(7):935-954.

[Boland et al., 2006] Boland, T., Xu, T., Damon, B., and Cui, X. (2006). Application of inkjet printing to tissue engineering. Biotechnol. J., 1:910-917.

[Bostwick et al., 2014] Bostwick, J. B., Shearer, M., and Daniels, K. E. (2014). Elastocapillary deformations on partially-wetting substrates: rival contact-line models. Soft Matter, 10(37):7361-7369.

[Cao et al., 2014] Cao, Z., Stevens, M. J., and Dobrynin, A. V. (2014). Adhesion and wetting of nanoparticles on soft surfaces. Macromolecules, 47(9):3203-3209.

[Chung, 2011] Chung, S. e. a. (2011). Inkjet-printed stretchable silver electrode on wave structured elastomeric substrate. Appl. Phys. Lett., 98:2011-2014.

[Ciarletta, 2013] Ciarletta, P. (2013). Surface instability of a gel disc in swelling. The European Physical Journal E, 36(2):1-4.

[De Gennes et al., 2013] De Gennes, P.-G., Brochard-Wyart, F., and Quéré, D. (2013). Capillarity and wetting phenomena: drops, bubbles, pearls, waves. Springer Science \& Business Media.

[Dervaux and Ben Amar, 2011] Dervaux, J. and Ben Amar, M. (2011). Buckling condensation in constrained growth. J. Mech. Phys. Solids, 59:538-560.

[Dervaux et al., 2011] Dervaux, J., Couder, Y., Guedeau-Boudeville, M.-A., and Ben Amar, M. (2011). Shape transition in artificial tumors: From smooth buckles to singular creases. Phys. Rev. Lett, 107:018103.

[Dervaux and Limat, 2015] Dervaux, J. and Limat, L. (2015). Contact lines on soft solids with uniform surface tension: analytical solutions and double transition for increasing deformability. In Proc. $R$. Soc. A, volume 471, page 20140813. The Royal Society.

[Jerison et al., 2011] Jerison, E. R., Xu, Y., Wilen, L. A., and Dufresne, E. R. (2011). Deformation of an elastic substrate by a three-phase contact line. Physical review letters, 106(18):186103.

[Le Tallec, 1994] Le Tallec, P. (1994). Numerical methods for nonlinear three-dimensional elasticity. Handbook of numerical analysis, 3:465-622.

[Lester, 1961] Lester, G. (1961). Contact angles of liquids at deformable solid surfaces. Journal of Colloid Science, 16(4):315-326.

[Limat, 2012] Limat, L. (2012). Straight contact lines on a soft, incompressible solid. The European Physical Journal E, 35(12):1-13.

[Lubarda, 2012] Lubarda, V. A. (2012). Mechanics of a liquid drop deposited on a solid substrate. Soft Matter, 8(40):10288-10297.

[Lubbers et al., 2014] Lubbers, L. A., Weijs, J. H., Botto, L., Das, S., Andreotti, B., and Snoeijer, J. H. (2014). Drops on soft solids: free energy and double transition of contact angles. Journal of fluid mechanics, 747:R1.

[Madasu and Cairncross, 2004] Madasu, S. and Cairncross, R. A. (2004). Static wetting on flexible substrates: a finite element formulation. International journal for numerical methods in fluids, 45(3):301319 .

[Marchand et al., 2012a] Marchand, A., Das, S., Snoeijer, J. H., and Andreotti, B. (2012a). Capillary pressure and contact line force on a soft solid. Physical review letters, 108(9):094301. 
[Marchand et al., 2012b] Marchand, A., Das, S., Snoeijer, J. H., and Andreotti, B. (2012b). Contact angles on a soft solid: from youngs law to neumanns law. Physical review letters, 109(23):236101.

[Mora et al., 2011] Mora, S., Abkarian, M., Tabuteau, H., and Pomeau, Y. (2011). Surface instability of soft solids under strain. Soft Matter, 7(22):10612-10619.

[Mora et al., 2013] Mora, S., Maurini, C., Phou, T., Fromental, J.-M., Audoly, B., and Pomeau, Y. (2013). Solid drops: Large capillary deformations of immersed elastic rods. Physical review letters, 111(11):114301.

[Mora et al., 2010] Mora, S., Phou, T., Fromental, J.-M., Pismen, L. M., and Pomeau, Y. (2010). Capillarity driven instability of a soft solid. Physical review letters, 105(21):214301.

[Mora and Pomeau, 2015] Mora, S. and Pomeau, Y. (2015). Softening of edges of solids by surface tension. Journal of Physics: Condensed Matter, 27(19):194112.

[Nadermann et al., 2013] Nadermann, N., Hui, C.-Y., and Jagota, A. (2013). Solid surface tension measured by a liquid drop under a solid film. Proceedings of the National Academy of Sciences, 110(26):10541-10545.

[Park et al., 2014] Park, S. J., Weon, B. M., San Lee, J., Lee, J., Kim, J., and Je, J. H. (2014). Visualization of asymmetric wetting ridges on soft solids with x-ray microscopy. Nature communications, 5 .

[Pericet-Camara et al., 2009] Pericet-Camara, R., Auernhammer, G. K., Koynov, K., Lorenzoni, S., Raiteri, R., and Bonaccurso, E. (2009). Solid-supported thin elastomer films deformed by microdrops. Soft Matter, 5(19):3611-3617.

[Roman and Bico, 2010] Roman, B. and Bico, J. (2010). Elasto-capillarity: deforming an elastic structure with a liquid droplet. Journal of Physics: Condensed Matter, 22(49):493101.

[Rusanov, 1975] Rusanov, A. (1975). Theory of wetting of elastically deformed bodies. 1. deformation with a finite contact-angle. Colloid Journal of the USSR, 37(4):614-622.

[Shanahan, 1987a] Shanahan, M. (1987a). The influence of solid micro-deformation on contact angle equilibrium. Journal of Physics D: Applied Physics, 20(7):945.

[Shanahan and De Gennes, 1987] Shanahan, M. and De Gennes, P. (1987). Equilibrium of the triple line solid/liquid/fluid of a sessile drop. In Adhesion 11, pages 71-81. Springer.

[Shanahan, 1987b] Shanahan, M. E. (1987b). Equilibrium of liquid drops on thin plates; plate rigidity and stability considerations. The Journal of Adhesion, 20(4):261-274.

[Shuttleworth, 1950] Shuttleworth, R. (1950). The surface tension of solids. Proceedings of the physical society. Section A, 63(5):444.

[Sokuler, 2010] Sokuler, M. e. a. (2010). The softer the better: Fast condensation on soft surfaces. Langmuir, 26:1544-1547.

[Steigmann and Ogden, 1997] Steigmann, D. and Ogden, R. (1997). Plane deformations of elastic solids with intrinsic boundary elasticity. In Proceedings of the Royal Society of London A: Mathematical, Physical and Engineering Sciences, volume 453, pages 853-877. The Royal Society.

[Style et al., 2013a] Style, R. W., Boltyanskiy, R., Che, Y., Wettlaufer, J., Wilen, L. A., and Dufresne, E. R. (2013a). Universal deformation of soft substrates near a contact line and the direct measurement of solid surface stresses. Physical review letters, 110(6):066103.

[Style and Dufresne, 2012] Style, R. W. and Dufresne, E. R. (2012). Static wetting on deformable substrates, from liquids to soft solids. Soft Matter, 8(27):7177-7184. 
[Style et al., 2013b] Style, R. W., Hyland, C., Boltyanskiy, R., Wettlaufer, J. S., and Dufresne, E. R. (2013b). Surface tension and contact with soft elastic solids. Nature communications, 4.

[Taffetani and Ciarletta, 2015] Taffetani, M. and Ciarletta, P. (2015). Beading instability in soft cylindrical gels with capillary energy: Weakly non-linear analysis and numerical simulations. Journal of the Mechanics and Physics of Solids, 81:91-120.

[Unger et al., 2000] Unger, M. A., Chou, H.-P., Thorsen, T., Scherer, A., and Quake, S. R. (2000). Monolithic microfabricated valves and pumps by multilayer soft lithography. Science, 80:113-116.

[Weijs et al., 2013] Weijs, J. H., Andreotti, B., and Snoeijer, J. H. (2013). Elasto-capillarity at the nanoscale: on the coupling between elasticity and surface energy in soft solids. Soft Matter, 9(35):84948503.

[White, 2003] White, L. R. (2003). The contact angle on an elastic substrate. 1. the role of disjoining pressure in the surface mechanics. Journal of colloid and interface science, 258(1):82-96.

[Xu et al., 2014] Xu, X., Jagota, A., and Hui, C.-Y. (2014). Effects of surface tension on the adhesive contact of a rigid sphere to a compliant substrate. Soft Matter, 10(26):4625-4632.

[Xu et al., 2013] Xu, X., Jagota, A., Peng, S., Luo, D., Wu, M., and Hui, C.-Y. (2013). Gravity and surface tension effects on the shape change of soft materials. Langmuir, 29(27):8665-8674.

[Yu, 2012] Yu, Y.-s. (2012). Substrate elastic deformation due to vertical component of liquid-vapor interfacial tension. Applied Mathematics and Mechanics, 33:1095-1114.

[Yu and Zhao, 2009] Yu, Y.-S. and Zhao, Y.-P. (2009). Deformation of pdms membrane and microcantilever by a water droplet: Comparison between mooney-rivlin and linear elastic constitutive models. Journal of colloid and interface science, 332(2):467-476. 